Nonconvergence of formal integrals: II. Improved estimates for the optimal order of truncation

This article has been downloaded from IOPscience. Please scroll down to see the full text article.

2004 J. Phys. A: Math. Gen. 3710831

(http://iopscience.iop.org/0305-4470/37/45/008)

View the table of contents for this issue, or go to the journal homepage for more

Download details:

IP Address: 159.149.103.9

The article was downloaded on 04/06/2013 at $17: 14$

Please note that terms and conditions apply. 


\title{
Nonconvergence of formal integrals: II. Improved estimates for the optimal order of truncation
}

\author{
C Efthymiopoulos ${ }^{1}$, A Giorgilli ${ }^{2}$ and G Contopoulos ${ }^{1}$ \\ ${ }^{1}$ Research Center for Astronomy, Academy of Athens, Anagnostopoulou 14, 10673 Athens, \\ Greece \\ ${ }^{2}$ Department of Applied Mathematics, University of Milano Bicocca, via Bicocca degli \\ Arcimboldi 8, 20126 Milano, Italy
}

Received 30 March 2004, in final form 17 September 2004

Published 28 October 2004

Online at stacks.iop.org/JPhysA/37/10831

doi:10.1088/0305-4470/37/45/008

\begin{abstract}
We investigate the asymptotic properties of formal integral series in the neighbourhood of an elliptic equilibrium in nonlinear 2 DOF Hamiltonian systems. In particular, we study the dependence of the optimal order of truncation $N_{\text {opt }}$ on the distance $\rho$ from the elliptic equilibrium, by numerical and analytical means. The function $N_{\text {opt }}(\rho)$ determines the region of Nekhoroshev stability of the orbits and the time of practical stability. We find that the function $N_{\text {opt }}(\rho)$ decreases by abrupt steps. The decrease is roughly approximated with an average power law $N_{\text {opt }}=O\left(\rho^{-a}\right)$, with $a \simeq 1$. We find an analytical explanation of this behaviour by investigating the accumulation of small divisors in both the normal form algorithm via Lie series and in the direct construction of first integrals. Precisely, we find that the series exhibit an apparent radius of convergence that tends to zero by abrupt steps as the order of the series tends to infinity. Our results agree with those obtained by Servizi G et al (1983 Phys. Lett. A 95 11) for a conservative map of the plane. Moreover, our analytical considerations allow us to explain the results of our previous paper (Contopoulos G et al 2003 J. Phys. A: Math. Gen. 36 8639), including in particular the different behaviour observed for low-order and higher order resonances.
\end{abstract}

PACS numbers: $05.45 .-\mathrm{a}, 45.10 .-\mathrm{b}$

\section{Introduction}

Formal integrals and normal forms in the neighbourhood of an elliptic equilibrium are wellstudied tools of the canonical perturbation theory. The interest in formal series stems from the fact that despite their divergence, demonstrated by Siegel (1941) as the effect of the accumulation of the so-called small divisors, such series have asymptotic properties which 
render them useful in a number of applications. The remainder of a formal series at order $r$ can be estimated as $R_{r} \sim r ! \rho^{r}$, where $\rho$ is the distance from the elliptic point (e.g. Giorgilli (1999)). For any fixed $\rho, \lim _{r \rightarrow \infty} R_{r}=\infty$. However, for $\rho$ small enough, $R_{r}$ initially decreases, as $r$ increases, and it can go close to zero before it starts increasing, for $r$ still larger. A particular example, for $\rho=0.001$, is given in Poincaré's Méthodes Nouvelles (Poincaré 1892). If we truncate the series at order $r=N_{\text {opt }}$ where the remainder is minimal, then, provided that this remainder is small, the truncated formal series induce a dynamics quite close to the dynamics of the true system for very long times. In particular, the behaviour of the functions $R_{r}(\rho)$ and $N_{\text {opt }}(\rho)$ allows us to prove a Nekhososhev (1977)-like result for the approximate constancy of formal integrals, namely that the variations of such integrals remain bounded over times exponentially long in the inverse of $\rho$. Furthermore, truncated expressions of the formal integrals are known to reproduce theoretically, with great accuracy, the invariant curves corresponding to invariant KAM tori on a Poincaré surface of section (Contopoulos and Moutsoulas 1965, Gustavson 1966, Kaluza and Robnik 1992, Contopoulos et al 2003). In this respect, the limit $N_{\text {opt }} \rightarrow 0$ provides interesting information on the dynamics, since it is connected with the phenomenon of breakdown of the invariant tori and with the introduction of a large degree of chaos in phase space. Such properties of the formal series render them useful in a wide spectrum of applications, ranging from atomic phenomena (e.g. Eckhardt (1986)) up to the scales of the solar system and of galactic dynamics (see Contopoulos (2002) for a review).

Now, the relation $R_{r} \sim r ! \rho^{r}$ is just a rough representation of the true function $R_{r}(\rho)$ the details of which depend crucially on a number of factors such as the dimension of the system and the number theoretical properties of the frequency ratios of the associated linearized system around the elliptic equilibrium. The purpose of our study is exactly to determine the detailed behaviour of the functions $R_{r}(\rho)$ and $N_{\text {opt }}(\rho)$ both by analytical and numerical methods. The analytical framework used in our considerations below is well known (see Arnold (1985), Giorgilli (1999) and Haller (1999) for general references). In particular, the question of the optimal exponents in Nekhoroshev-type estimates near elliptic equilibria has been investigated by Lochak (1992), Fassò et al (1998) and Niederman (1998). On the other hand, numerical results connected to our study are only sporadic in the literature (Servizi et al 1983, Kaluza and Robnik 1992, Morbidelli and Giorgilli 1997).

In our previous paper (Contopoulos et al (2003), hereafter paper I), we studied numerically the asymptotic properties of formal series representing approximate first integrals of motion in the neighbourhood of an elliptic equilibrium in 2 DOF Hamiltonian systems of the form

$$
H \equiv \frac{1}{2}\left(\dot{x}^{2}+\dot{y}^{2}+\omega_{1}^{2} x^{2}+\omega_{2}^{2} y^{2}\right)+H_{3}(x, y)
$$

for various models of the coupling term $H_{3}(x, y)$. We considered only resonance cases, i.e. $\omega_{1} / \omega_{2}=$ rational. In particular, we considered the lowest possible resonance $1: 1$ as well as a higher order resonance $4: 3$. The main focus was to define numerically the optimal order of truncation $N_{\text {opt }}$ of the formal series as a function of the distance $\rho$ from the elliptic equilibrium. The optimal order is defined as the order at which the time variations $\Delta I$ of the approximate integral $I$, defined by truncating the formal series at order $N=N_{\text {opt }}$, are minimal.

In the present paper, we develop a framework to understand the behaviour of the function $N_{\text {opt }}(\rho)$ from an analytical point of view. But before that, we study the problem further from the numerical point of view, by considering various non-resonant cases in which the frequencies satisfy a diophantine condition. As shown in the following sections, the main ingredients of the problem under study have indeed been found already in the non-resonant case.

Our findings for the non-resonant case represent a shift from the traditional picture for the accumulation of small divisors in the terms produced by successive iterations of the 
formal procedure. A correct description of the accumulation of small divisors is given more easily in terms of the normal form construction with Lie series. The latter will be our main tool for analytic considerations. From the numerical point of view, however, the analysis is facilitated by the use of a direct method of construction of the formal integrals due to Whittaker (1916, 1937), Cherry 1924) and Contopoulos (1960). We use this method in our numerical analysis and explain also how the results found with normal forms translate to the results found with formal integrals by the direct method.

An essential point considered in the present paper is the dependence of the function $N_{\text {opt }}(\rho)$ on the number theoretical properties of the ratio $\omega_{1} / \omega_{2}$. We emphasize that the diophantine conditions used in almost all forms of canonical perturbation theory provide optimal estimates of the smallness of the divisors appearing in a formal series only at some particular orders. We recall that a diophantine condition for the frequencies is a relation of the form

$$
|m \cdot \omega|=\left|m_{1} \omega_{1}+m_{2} \omega_{2}+\cdots+m_{n} \omega_{n}\right| \geqslant \frac{\gamma}{|m|^{\tau}}
$$

where $\gamma$ is a positive constant, $m_{i}$ are integers, $|m|=\sum_{i=1}^{n}\left|m_{i}\right|$, and $\tau \geqslant n-1$, where $n$ is the number of degrees of freedom. We state that (2) provides an optimal estimate of the smallness of the divisors at order $|m|$ if there are $m_{i}$ with $|m|=\sum_{i=1}^{n}\left|m_{i}\right|$ such that for these particular $m_{i}$ relation (2) is an equality. The corresponding divisor $|m \cdot \omega|$ will be called a 'diophantine divisor'. In the 2 DOF case, the diophantine condition (2) is optimal only at some specific orders defined by the simple continued fraction representation of the ratio $\omega_{1} / \omega_{2}$. For all other orders relation (2) is a strict inequality. This is a crucial remark; its consequences are examined in detail below.

The paper is organized as follows. Section 2 presents the numerical results for the function $N_{\text {opt }}(\rho)$ in the non-resonance case. Three cases are considered, which correspond to different degrees of 'nobleness' of the number $\omega_{1} / \omega_{2}$. Section 3 summarizes first the old analytical framework in terms of both the direct and normal form methods. Then it presents the new framework that leads to a theoretical prediction of the function $N_{\text {opt }}(\rho)$ that agrees with the function found numerically in section 2. Section 4 discusses the resonance case from a theoretical framework that allows us to interpret the results found numerically in our previous paper. Finally, section 5 contains the main conclusions of the present study.

\section{Numerical determination of $N_{\text {opt }}(\rho)$}

Following a trivial normalization, we consider the Hamiltonian

$$
H \equiv \frac{\omega_{1}}{2}\left(x^{2}+p_{x}^{2}\right)+\frac{\omega_{2}}{2}\left(y^{2}+p_{y}^{2}\right)+0.1\left(x^{3}+x^{2} y+x y^{2}+y^{3}\right)
$$

for various non-resonant values of the real frequencies $\omega_{1}, \omega_{2}$ satisfying a diophantine condition of the form (2). It should be stressed that an analytic Hamiltonian perturbation is given, in general, as an expansion to all orders $H_{3}+H_{4}+\cdots$. Thus, the Hamiltonian (3) is special in the sense that it contains only an $\mathrm{H}_{3}$ term. Nevertheless, as will be shown below, this simple model suffices to show the mechanisms of accumulation of small divisors in the formal series. In fact, as demonstrated by Morbidelli and Giorgilli (1997), higher order terms with an amplitude small enough to preserve the analyticity of the Hamiltonian are born at successive steps of the normalization process even if such terms are not present in the original Hamiltonian. 


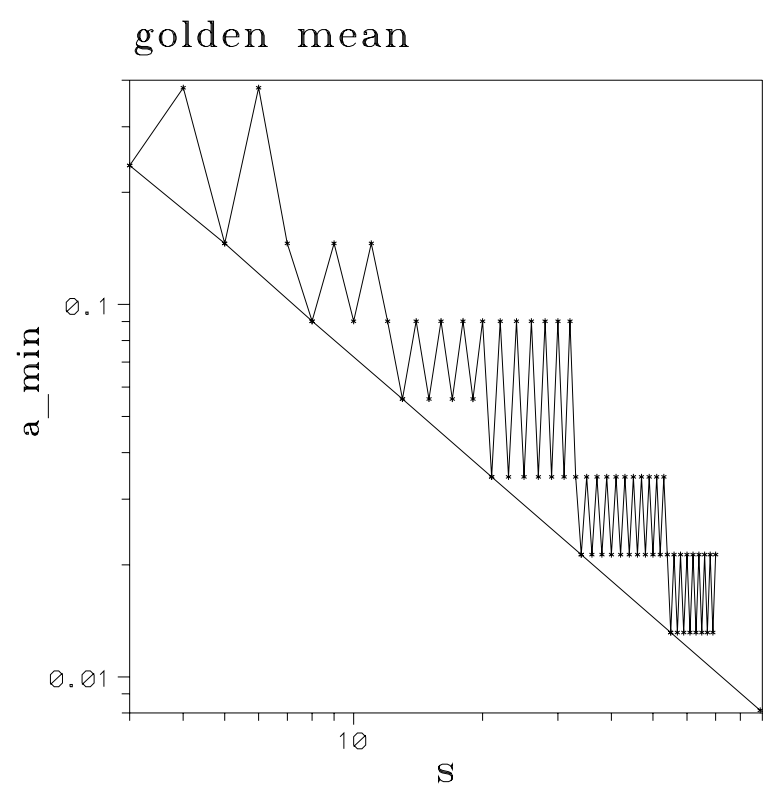

Figure 1. The minimum divisor $a_{\min }$ that can appear in a formal series at order $s$ as a function of $s$ for the frequencies $\omega_{1}=1, \omega_{2}=(\sqrt{5}-1) / 2$ (golden mean).

\section{1. $\omega_{2} / \omega_{1}=$ golden mean}

We consider first the frequency ratio $\omega_{2} / \omega_{1}=(\sqrt{5}-1) / 2=0.618 \ldots$ by letting the frequencies to be $\omega_{1}=1$ and $\omega_{2}=(\sqrt{5}-1) / 2$. One finds that (figure 1 )

$$
a_{s} \geqslant \frac{0.713}{|s|}
$$

where

$$
a_{s}=\min |m \cdot \omega|
$$

for all $m \equiv\left(m_{1}, m_{2}\right)$ with $s \geqslant|m|=\left|m_{1}\right|+\left|m_{2}\right|,|m|$ having the same parity as $s$. According to standard theory, the divisors $|m \cdot \omega|$ with the above restrictions are the only ones that may appear in the formal construction at order $s$.

The solid line in figure 1 represents the optimal relation $a_{s}=\gamma / s^{\tau}$. As expected from the theory of continued fractions, the diophantine condition (2) is an equality only at those orders $s=\left|m_{1}\right|+\left|m_{2}\right|$ where there are $m_{1}, m_{2}$ for which $m_{1} / m_{2}=-q_{n} / p_{n}$, the $n$th rational truncation of the continued fraction representation $\omega_{2}=[1,1,1, \ldots]$.

A second formal integral $\Phi$ can be found by solving recursively the equations

$$
D_{\omega} \Phi_{s}=-\left\{\Phi_{s-1}, H_{3}\right\}, \quad D_{\omega} \cdot=\left\{\cdot, H_{2}\right\}
$$

where $H_{2}, H_{3}$ are the terms of second and third degree of the Hamiltonian respectively. We start with $\Phi_{2}=\frac{1}{2}\left(p_{x}^{2}+x^{2}\right)$. Then, equation (6) can be solved order by order and it ensures that the quantity $\Phi=\Phi_{2}+\Phi_{3}+\cdots$ has zero Poisson bracket with the Hamiltonian, i.e. it is a formal integral of motion.

This 'direct' method of calculating the integrals step by step via equation (6) (Whittaker 1916, 1937, Cherry 1924, Contopoulos 1960) is as old as the Birkhoff-Gustavson method (Birkhoff 1927, Gustavson 1966) of determining the formal integrals via normal forms. Its extension to deal with resonance cases was given by Contopoulos (1963). In our previous 
paper, we implemented the normal form method via a variant of Lie series (Giorgilli 1979); however, this method introduces a technical complication: it gives integrals as linear combinations of powers of the actions and this affects the convergence properties even in the case of integrable systems (paper I). This can be understood with the help of the following elementary example. Consider the one-dimensional Hamiltonian

$$
H \equiv \frac{1}{2}\left(p^{2}+x^{2}\right)+x^{3} .
$$

In this case, we expect to find one formal integral equal to the Hamiltonian, i.e. the only existing integral. However, if we calculate the formal integral with the Birkhoff normal form (e.g. with Giorgilli's (1979) program), we find that

$$
I=\frac{1}{2}\left(p^{2}+x^{2}\right)+x^{3}+\frac{15}{16}\left(x^{4}+p^{4}+2 x^{2} p^{2}\right)+\frac{15}{4}\left(x^{5}+x^{3} p^{2}\right)+\cdots
$$

i.e. an infinite series in the canonical variables. This is due to the fact that the normal form, i.e. the Hamiltonian, as a function of the action $I$ reads

$$
H=I-\frac{15}{4} I^{2}-\frac{705}{16} I^{3}+\cdots
$$

i.e. it is a series in the action variable. The series (8) comes from the inversion of the series (9), namely

$$
I=H+\frac{15}{4} H^{2}+\frac{1155}{16} H^{3}+\cdots
$$

which gives us only a finite radius of convergence along any direction in phase space. For example, if we calculate the radius of convergence of the series $I$ with a d'Alembert or a Cauchy criterion along the particular direction $p=0$, we find $R_{c}=0.186$. This kind of problem is well known (e.g. Eckhardt (1986), Wood and Ali (1987)).

On the other hand, if we calculate a formal integral with the direct method in the case of the Hamiltonian (7), starting with $\Phi_{2}=\frac{1}{2}\left(x^{2}+p^{2}\right)$ we find $\Phi_{3}=x^{3}$ and $\Phi_{s}=0$ for $s>3$, i.e. we recover the Hamiltonian integral without any higher order terms. It is possible to recover the same integral $\Phi$ using the normal form method, by taking appropriate power series of the integrals found by the normal form, but the algorithm to calculate the higher order terms is complicated.

The optimal order of truncation of the formal integral can be defined numerically by the method used in paper I. Namely, we consider the time variations $\left|\Delta I_{N}\right|$ of the approximate integral $I_{N}$ corresponding to the $N$ th order of truncation

$$
I_{N}=\Phi_{2}+\Phi_{3}+\cdots+\Phi_{N}
$$

calculated numerically for a sufficient time of integration and for different initial conditions along a particular direction of phase space (see paper I for details). Then we find the order at which the variation $\Delta I_{N}$ is minimum. In the calculations throughout the present paper, $\Phi$ is expressed as a polynomial series in the complex canonical variables $\left(q_{i}, p_{i}\right), i=1$, 2 , with

$$
q_{i}=\frac{X_{i}-\mathrm{i} P_{i}}{\sqrt{2}}, \quad p_{i}=\frac{P_{i}-\mathrm{i} X_{i}}{2}
$$

with $X_{1} \equiv x, X_{2} \equiv y, P_{1} \equiv p_{x}, P_{2} \equiv p_{y}$. The solution of the recursion relation (6) in the variables $\left(q_{i}, p_{i}\right)$ reduces to the solution of a simple diagonal linear system of equations for the unknown coefficient of each term of $\Phi_{s}$. This is because any monomial $q_{1}^{k_{1}} p_{1}^{l_{1}} q_{2}^{k_{2}} p_{2}^{l_{2}}$ is an eigenfunction of the linear operator $D_{\omega}$, appearing in the lhs of equation (6). Namely, we have

$$
D_{\omega} q_{1}^{k_{1}} p_{1}^{l_{1}} q_{2}^{k_{2}} p_{2}^{l_{2}}=\mathrm{i}\left(\left(k_{1}-l_{1}\right) \omega_{1}+\left(k_{2}-l_{2}\right) \omega_{2}\right) q_{1}^{k_{1}} p_{1}^{l_{1}} q_{2}^{k_{2}} p_{2}^{l_{2}}
$$

or, in compact notation, $D_{\omega} q^{k} p^{l}=\mathrm{i}[(k-l) \cdot \omega] q^{k} p^{l}$. 
(a)

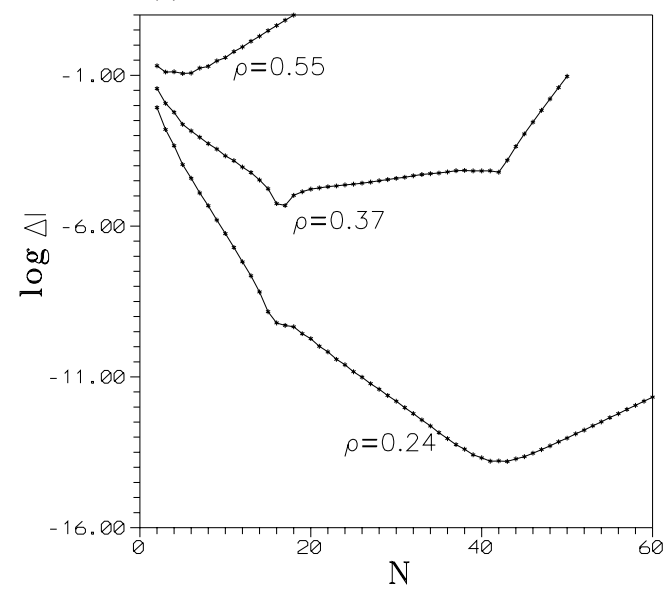

(b)

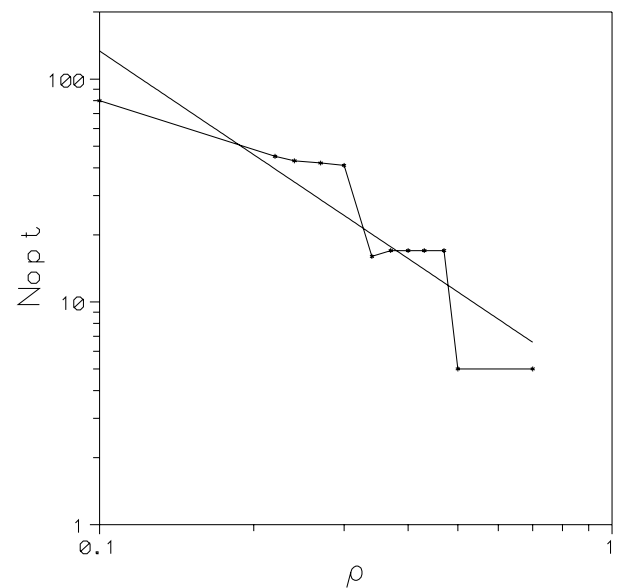

Figure 2. (a) The variations $\Delta I$ of the numerical value of the formal integral $I_{N}$ truncated at order $N$, as a function of $N$, for three orbits with different initial distances $\rho$ from the elliptic equilibrium. The Hamiltonian is given by equation (3) and the frequencies are $\omega_{1}=1, \omega_{2}=$ $(\sqrt{5}-1) / 2$. (b) The optimal order of truncation $N_{\text {opt }}$, corresponding to the minima of the curves of figure $3(a)$, as a function of the initial distance $\rho$.

In the cases considered below we calculate the formal integral up to order 120 .

Figure 2(a) shows the variations $\left|\Delta I_{N}\right|$ as functions of the order of truncation $N$ for different initial distances $\rho$ along the direction $x=y$ and $p_{x}=p_{y}=0$. For all initial conditions, the variations $\left|\Delta I_{N}\right|$ initially decrease, indicating an apparent convergence of the formal integral. For initial conditions close to the origin $(\rho=0.24$ in figure $2(a)$ ) the variations for successive orders of truncation decrease down to the computer precision $\left(10^{-14}\right)$ so that $I_{N}$ for the optimal $N$ becomes almost a perfect integral. As $\rho$ increases the variations become larger in general. However, near the minimum they are still relatively small (e.g. $10^{-6}$ for $\rho=0.37$ ). Thus $I_{N}$ still represents an approximate integral. Only at large distances $\rho$, where chaos prevails, the variations $\left|\Delta I_{N}\right|$ become large (of order unity). Then the formal series no longer represents even an approximate integral.

The optimal order $N_{\text {opt }}$ for given initial distance $\rho$ is defined by the minimum of the curve $\left|\Delta I_{N}\right|(N)$. If we find $N_{\text {opt }}$ for various distances $\rho$ we define numerically the dependence of $N_{\text {opt }}$ on $\rho$. This is shown in figure $2(b)$, on a logarithmic scale. The function $N_{\text {opt }}(\rho)$ is characterized by approximate plateaux (roughly at orders 5, 15 and 50) which are connected by abrupt steps. Around $\rho=0.1$ the optimal order changes again abruptly, but the new optimal order cannot be defined with accuracy because the corresponding variations $\Delta I$ are smaller than the computer precision. If we plot a power law fitting (a straight line in figure $2(b)$ ) for the curve $N_{\text {opt }}$, we find an average power law

$$
N_{\text {opt }}=3.8 \rho^{-1.5}
$$

for $0.1 \leqslant \rho \leqslant 0.6$.

An independent test of the dependence of the optimal order on the distance $\rho$ can be done by calculating the norm of the remainder function of the series $\Phi$. The time derivative $\dot{I}_{N}$ at any truncation $N$ differs from zero by

$$
\dot{I}_{N}=\left[\Phi_{N}, H_{3}\right] \equiv R_{N+1}
$$


(a)

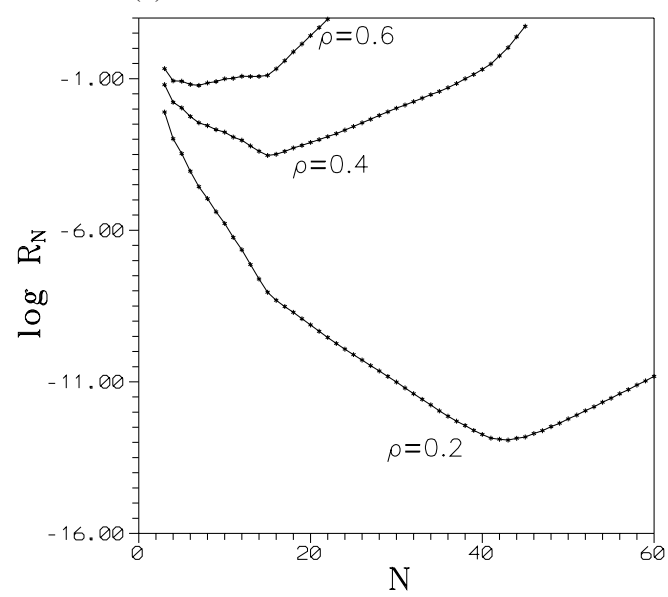

(b)

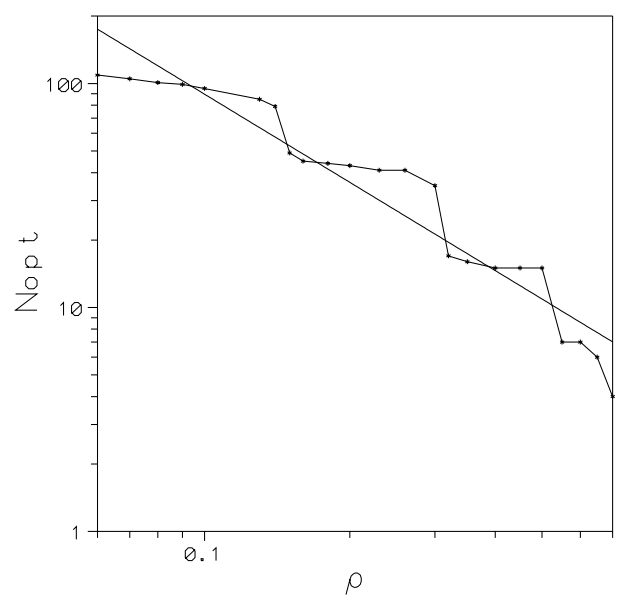

Figure 3. (a) The norm of the remainder $R_{N}$ (equations (16) and (19)) as a function of the order $N$, for different distances $\rho$ from the elliptic equilibrium, for the same Hamiltonian and frequencies as in figure 2. (b) The optimal order of truncation $N_{\text {opt }}$ as a function $\rho$ by the remainder criterion, i.e. by calculating the minima of the curves of figure $3(a)$.

and it will be referred to as the $(N+1)$-order remainder. We then calculate an appropriate norm of the remainder at distance $\rho$. Let

$$
R_{N}=\sum_{k+l=N} R_{k l} q^{k} p^{l}
$$

be a $N$ th degree polynomial, $k \equiv\left(k_{1}, k_{2}\right), l \equiv\left(l_{1}, l_{2}\right), k+l=k_{1}+k_{2}+l_{1}+l_{2}$ and $R_{k l}$ are the constant polynomial coefficients. We define the polynomial norm as

$$
\left\|R_{N}\right\|=\sum_{k+l=N}\left|R_{k l}\right|
$$

and the norm at distance $\rho$ as

$$
\left\|R_{N}\right\|_{\rho}=\rho^{N} \sum_{k+l=N}\left|R_{k l}\right|
$$

Figure 3(a) shows the norm $\left\|R_{N}\right\|_{\rho^{\prime}}$ as a function of the order $N$ at various distances $\rho^{\prime}$. The curves in this figure are qualitatively very similar to the curves of figure $2(a)$. The minimum of each of the curves of figure 3(a) defines an optimal order of truncation $N_{\text {opt }}$ which minimizes the norm $\left\|R_{N}\right\|_{\rho^{\prime}}$. Due to equation (15) this is the order at which the time variations of the approximate integral $I_{N}$ are expected to be minimal.

If we plot $N_{\text {opt }}$ defined by the minimum of the norm $\left\|R_{N}\right\|_{\rho^{\prime}}$ versus $\rho^{\prime}$ we obtain the curve of figure $3(b)$. This compares very well (except for a constant logarithm, $\log \rho_{*}$ ) with the corresponding curve of figure $2(b)$. There are three plateaux at roughly the same orders as in figure $2(b)$. There is one more plateau at $N \simeq 100$ which does not appear in figure $2(b)$ because, as explained above, for this plateau, the numerical variations $\Delta I$ along the orbits integrated numerically are smaller than the computer accuracy so that the plateau cannot be defined numerically with precision. A best fit power law to the curve of figure $3(b)$ is

$$
N_{\text {opt }}=4.4 \rho^{\prime-1.3} \text {. }
$$

The power laws found numerically (equation (14)) and by the remainder criterion (equation (19)) turn out to have not very different exponents, taking into account that they are 

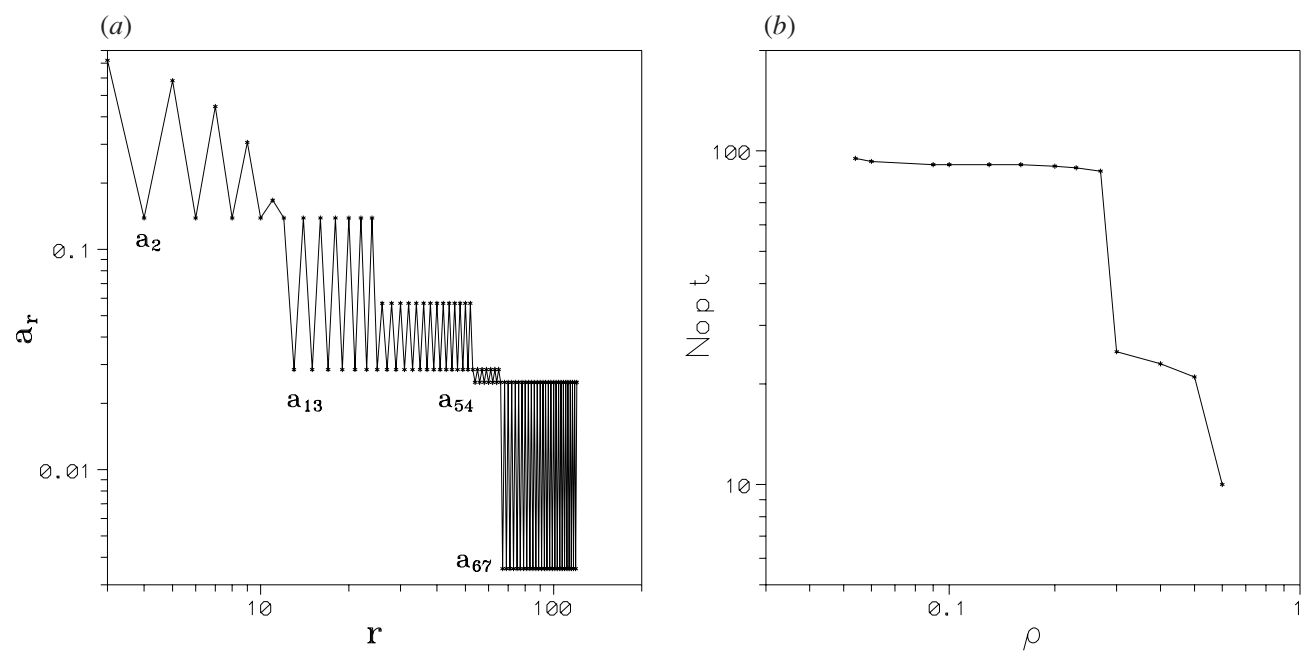

Figure 4. (a) Same as in figure 1, for the frequencies $\omega_{1}=1, \omega_{2}=12^{1 / 4}-1$. Newborn small divisors appear at orders $r=2,13,54$ and 67. (b) Same as in figure 3(b), for the frequencies $\omega_{1}=1, \omega_{2}=12^{1 / 4}-1$.

evaluated with quite few data. Also, the plateaux found in both cases correspond to about the same values of $N$. We conclude that the variations of the norm of the remainder $\left\|R_{N}\right\|_{\rho^{\prime}}$ provide a good estimate of the dependence of the numerical variations $\left|\Delta I_{N}\right|$ on the distance $\rho$ from the origin.

\section{2. $\omega_{2} / \omega_{1}=12^{1 / 4}-1$}

The purpose of the second choice of rotation number $\omega_{2} / \omega_{1}=12^{1 / 4}-1$ is to study the isolated phenomena introduced in the series when just one new diophantine divisor is introduced. The continued fraction of $\omega_{2} / \omega_{1}$ is $12^{1 / 4}-1=[1,6,4,1,7, \ldots]$. The corresponding rational truncations are $1 / 1,6 / 7,25 / 29,31 / 36,242 / 281, \ldots$ Thus new diophantine divisors are introduced at orders $2,13,54,67,523$, being $a_{2}=0.13879 \ldots$, $a_{13}=0.028468 \ldots, a_{54}=0.024918 \ldots, a_{67}=0.003549 \ldots, a_{523}=0.000069 \ldots$ respectively. As shown in figure $4(a)$, the divisor $a_{13}$ is the minimum divisor that appears in the formal series (at every second order) for $41=54-13$ order steps. The divisor $a_{54}$ is not so important because its value is close to the value of the divisor $a_{13}$. The next important divisor is $a_{67}$, which is the minimum divisor from order 67 up to 120 , which is our last order of calculation of the formal integral. Thus there are two leading divisors, namely $a_{13}$ and $a_{67}$ and the interval of steps between them is long enough so that the phenomena introduced by each of these divisors are well isolated.

If we calculate the optimal order of truncation $N_{\mathrm{opt}}$ as a function of the distance $\rho$ from the origin, with the remainder criterion, we find the curve shown in figure $4(b)$. This curve has two plateaux at roughly the orders 91 and 23. The transition from one plateau to the other is abrupt. In this case drawing an average power law has no meaning.

Similar plateaux were found in figure 13 of paper I, in the resonant case $\omega_{1} / \omega_{2}=4 / 3$. On the other hand, the function $N_{\text {opt }}(\rho)$ appeared to be rather smooth in the case of the $1: 1$ resonance (figure 10 of paper 1 , for the same Hamiltonian with $\omega_{1}=\omega_{2}=1$ ). These differences are explained in section 4 below. 


\section{Accumulation of small divisors}

The purpose of this section is to demonstrate the way by which small divisors accumulate in the terms of the series as well as to estimate the overall growth of the size of the terms of the series due to the accumulation of divisors. We consider first the non-resonant case. The resonant case will be considered in section 4 .

\subsection{Summary of steps and results}

In order to guide the reader through the rather technical details of the subsections below, we provide a summary of steps and results which are contained in these subsections.

Subsection 3.2.1. We give the definitions of the Lie generating function and of the Lie series method of calculation of the normal form around an elliptic equilibrium. We also define action-angle $(J, \phi)$ variables suitable for the systems under study.

Subsection 3.2.2. We examine the way by which particular Fourier modes $a(J) \exp (\mathrm{i} k \cdot \phi)$, which appear for the first time in the generating function $\chi_{r}$ (i.e. Fourier terms of order $r$ with amplitude of order $r / 2$ in the actions), propagate in the subsequent terms of the generating function $\chi_{r+2}, \chi_{r+4}$, etc. The term 'propagate' means that each Fourier term of the form $a(J) \exp (\mathrm{i} k \cdot \phi)$, appearing in $\chi_{r}$, produces a number of new terms in the generating function at subsequent orders, which correspond to the same or a different Fourier mode. By using a well-known schematic representation of the propagation algorithm for Lie series, known as the Lie triangle, we reach the following result:

Each Fourier term $a(J) \exp (\mathrm{i} k \cdot \phi)$, appearing in the generating function $\chi_{r}$ produces terms of Fourier modes $a^{\prime}(J) \exp \left(\mathrm{i} k^{\prime} \cdot \phi\right)$ in the generating function at subsequent orders according to the following rules:

(a) Only terms of the same Fourier mode, i.e. $k^{\prime}=k$ are produced in the generating function up to order $2 r$.

(b) Beyond order $2 r$, terms of both the same and of different Fourier modes are produced in the generating function.

The sequences of terms of the same Fourier mode generated at every second order are called repetitions. We demonstrate that the growth of the size of terms of a repeated Fourier mode $a(J) \exp (\mathrm{i} k \cdot \phi)$ is geometrical, with a fixed ratio.

Subsection 3.2.3. We specify the terms (Fourier modes) which produce the dominant contribution to the overall size of the generating function $\chi_{r}$. We conclude that the growth of the overall size of $\chi_{r}$ is 'piecewise geometrical', i.e., it appears to be geometrical for several orders, with a fixed ratio. However, this ratio increases abruptly at some specific orders corresponding to the appearance of new small divisors, i.e., the radius of convergence decreases by abrupt steps and it goes to zero as $r \rightarrow \infty$. Numerical examples of this behaviour are given (figure 5).

Subsection 3.3.1. We sketch some old estimates (Giorgilli 1988) about the growth of the series terms in formal integrals calculated by the 'direct' method.

Subsection 3.3.2. We explain how repetitions appear in the direct method, by defining paths of successive divisors which appear in the terms at successive orders. Thus we recover essentially the growth factor for terms in repetition paths given by equation (38).

Subsection 3.3.3. We examine the growth of terms in non-repetition paths and demonstrate that it cannot be faster than the growth of terms in repetition paths.

Subsection 3.3.4. We analyse the phenomenon of 'delays', i.e., the difference between the order at which new Fourier modes appear, and the order at which these Fourier modes become dominant in the series. 

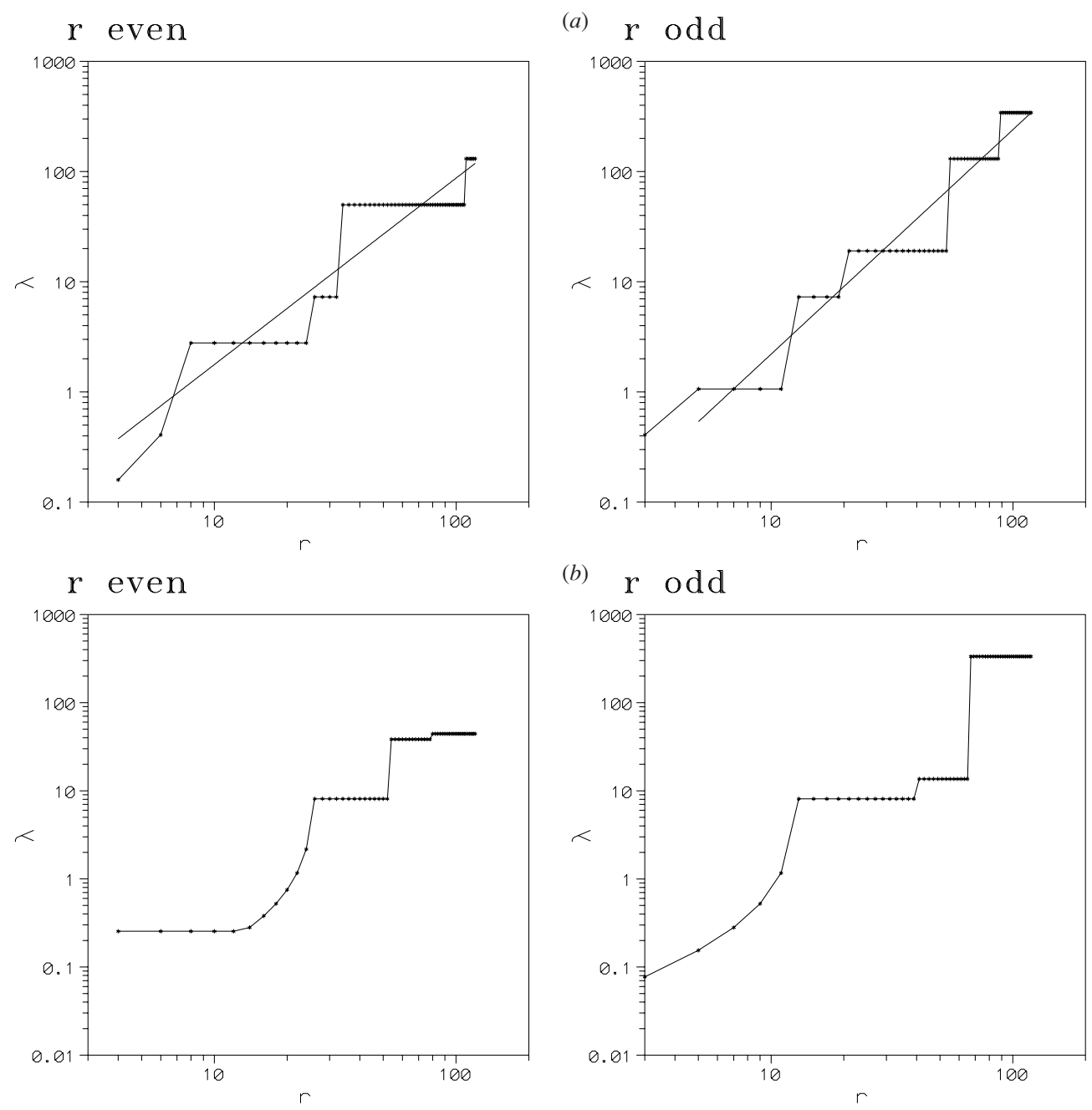

(b) $\mathrm{r}$ odd

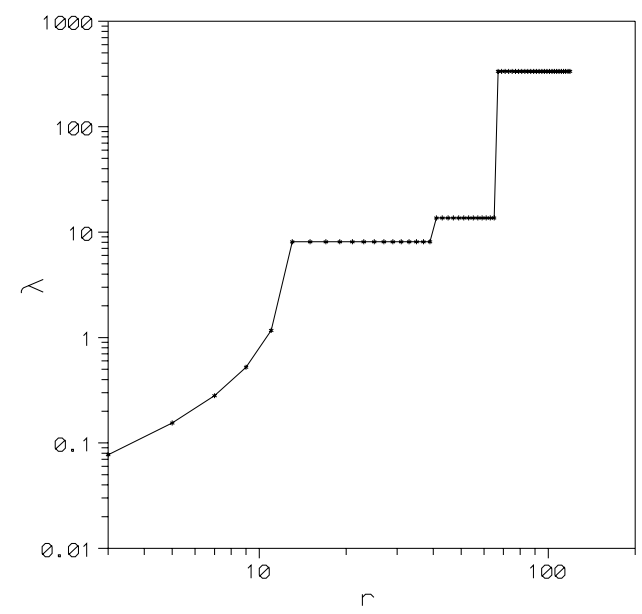

Figure 5. The ratio $\lambda_{r}$ as given by equation (38), for $r$ even and odd. (a) $\omega_{1}=1, \omega_{2}=(\sqrt{5}-1) / 2$; (b) $\omega_{1}=1, \omega_{2}=12^{1 / 4}-1$.

Subsection 3.4. Numerical examples are given which demonstrate the phenomena described in the previous subsections.

\subsection{Accumulation of small divisors by the normal form}

3.2.1. Definitions. We will use the Lie method of calculation of the normal form (Hori 1966, Deprit 1969). We recall that the Lie method introduces canonical transformations as mappings $q, p \rightarrow q^{\prime}, p^{\prime}$ induced by the Hamiltonian flow for $t=1$ of a generating function $\chi$. Namely, any function $F(q, p)$ of the old canonical variables (including $q, p$ themselves) is transformed to a function $F\left(q^{\prime}, p^{\prime}\right)$ of the new canonical variables according to

$$
F\left(q^{\prime}, p^{\prime}\right)=\exp \left(L_{\chi}\right) F(q, p)
$$

where $L_{\chi}$ is the Poisson bracket operator of the function $\chi$. After $r$ successive canonical transformations with generating functions $\chi_{3}, \chi_{4}, \ldots, \chi_{r}$, where $\chi_{s}$ is of order $s$ in the 
canonical variables, the transformed Hamiltonian (denoted $H^{(r)}$ ) is brought to normal form up to order $r$, namely

$H^{(r)} \equiv \exp \left(L_{\chi_{r}}\right) \exp \left(L_{\chi_{r-1}}\right) \cdots \exp \left(L_{\chi_{3}}\right) H=Z_{2}+Z_{3}+Z_{4}+\cdots Z_{r}+h_{r+1}^{(r)}+\cdots$.

Note that with the notation above, $H^{(2)}$ corresponds to the original Hamiltonian, i.e., as if we acted on the original Hamiltonian with an identity transformation.

In the non-resonant case the functions $Z_{r}$ (of order $r$ ) depend only on the quantities $q_{i} p_{i}, i=1,2$, so that $Z_{r}$ is zero for $r$ odd.

The equation defining the normal form $Z_{r}$ is

$$
Z_{r}=\left\langle h_{r}^{(r-1)}\right\rangle
$$

where $\left\langle h_{r}^{(r-1)}\right\rangle$ denotes all terms of $h_{r}^{(r-1)}$ which belong to the kernel of the operator $D_{\omega}$. On the other hand, the generating function $\chi_{r}$ is determined by

$$
D_{\omega} \chi_{r}-h_{r}^{(r-1)}+Z_{r}=0
$$

so that it contains all the terms of $h_{r}^{(r-1)}$ belonging to the range of the operator $D_{\omega}$, plus any arbitrary linear combination of terms of order $r$ belonging to the kernel of the operator $D_{\omega}$ (usually we set the coefficients of all such terms equal to zero). This method is well known in the literature (see Boccaletti and Pucacco (1999), chapter 8, for an instructive introduction).

We will consider furthermore the formal construction in action-angle variables $\left(J_{i}, \phi_{i}\right)$ defined by $q_{i}=\sqrt{J_{i}} \exp \left(\mathrm{i} \phi_{i}\right)$, i $p_{i}=\sqrt{J_{i}} \exp \left(-\mathrm{i} \phi_{i}\right)$. A polynomial term $q_{1}^{k_{1}} p_{1}^{l_{1}} q_{2}^{k_{2}} p_{2}^{l_{2}}$ is written as

$$
q_{1}^{k_{1}} p_{1}^{l_{1}} q_{2}^{k_{2}} p_{2}^{l_{2}}=\mathrm{i}^{l_{1}+l_{2}} J_{1}^{\frac{k_{1}+l_{1}}{2}} J_{2}^{\frac{k_{2}+l_{2}}{2}} \exp \left[\mathrm{i}\left(k_{1}-l_{1}\right) \phi_{1}+\mathrm{i}\left(k_{2}-l_{2}\right) \phi_{2}\right]
$$

where $r=k_{1}+l_{1}+k_{2}+l_{2}$. The kernel of the operator $D_{\omega}$ coincides thus with the functions which are independent of the angles, while the generating function $\chi$ will contain only fluctuating, i.e., angle-dependent terms. Note that a polynomial term of order $r$ corresponds to a term of order $r / 2$ in the actions and to a Fourier mode of order $|k-l| \equiv\left|k_{1}-l_{1}\right|+$ $\left|k_{2}-l_{2}\right|$ which is smaller than or equal to $r$, and of the same parity as $r$. This is relevant when we solve the equations (6), or (23), because the inverse operator $D_{\omega}^{-1}$ introduces divisors $a_{r}=(k-l) \cdot \omega$,

$$
D_{\omega}^{-1}\left[J^{\frac{r}{2}} \mathrm{e}^{\mathrm{i}(k-l) \cdot \phi}\right]=\frac{-\mathrm{i}}{(k-l) \cdot \omega} J^{\frac{r}{2}} \mathrm{e}^{\mathrm{i}(k-l) \cdot \phi} .
$$

The order $|k-l|$ of a Fourier mode $\exp (\mathrm{i}(k-l) \cdot \phi)$ will also be referred to as the order of the associated divisor $a_{r}=|(k-l) \cdot \omega|$. Thus the divisors appearing in equation (25) are of order less than or equal to $r$, and of the same parity as $r$. This means that a divisor which appeared for the first time at order $r_{0}$ may appear an infinite number of times in some terms of the formal series at all orders $r>r_{0}$ with the same parity as $r_{0}$.

3.2.2. Propagation of Fourier modes and accumulation of small divisors in the generating function. An implementation of the recursion scheme introduced by equations (22) and (23) reveals how small divisors accumulate in the generating function (and in the normal form). Assume that the normal form has been determined up to order $r-1$; thus we can determine the generating function $\chi_{r}$. The recursion in the subsequent step is given by the Lie triangle 


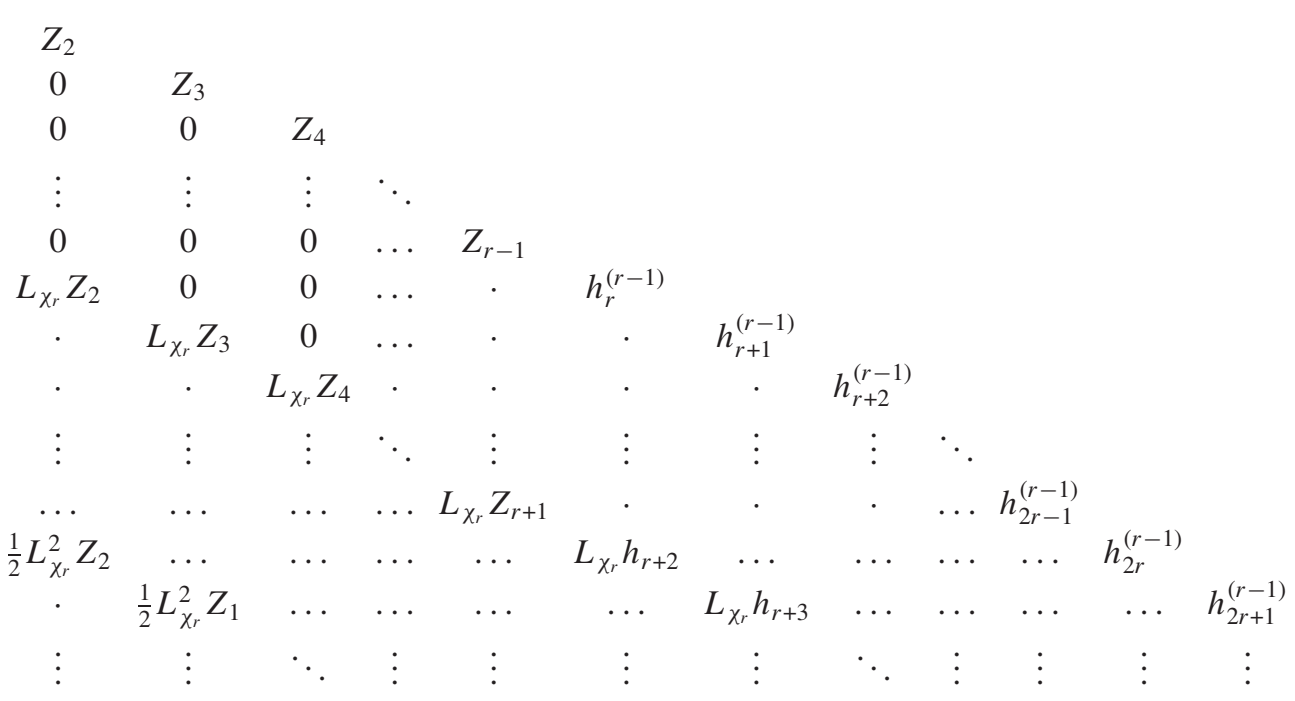

The upper diagonal contains the full Hamiltonian after $r-1$ transformations. The exponential operator $\exp \left(L_{\chi_{r}}\right)$ acts by columns, thus allowing us to calculate the whole triangle. The sum of the terms in the sth line of the triangle gives $h_{s}^{(r)}$. In particular, the $r$ th line gives the equation $Z_{r}=L_{\chi_{r}} Z_{2}+h_{r}^{(r-1)}$, by which $\chi_{r}$ is defined.

Since in the non-resonant case the normal form does not contain odd terms, we have $Z_{3}=0$ and $Z_{4} \neq 0$ (in general). Thus we have $h_{r+1}^{(r)}=h_{r+1}^{(r-1)}$ and $h_{r+2}^{(r)}=h_{r+2}^{(r-2)}+L_{\chi_{r}} Z_{4}$. In a similar way we find that $\chi_{r}$ affects only the terms of the Hamiltonian which have the same parity as $r$. Note also that any Fourier mode of $\chi_{r}$ remains unaltered by the operation $L_{\chi_{r}} Z_{4}$, because $Z_{4}$ contains only terms independent of the angles; this is the crucial remark in order to grasp how the small divisors accumulate.

Now, the generating function $\chi_{r+2}$ is determined by

$$
D_{\omega} \chi_{r+2}-h_{r+2}^{(r+1)}+Z_{r+2}=0
$$

and since $h_{r+2}^{(r+1)}=h_{r+2}^{(r)}$ we obtain

$$
D_{\omega} \chi_{r+2}-h_{r+2}^{(r)}+Z_{r+2}=0
$$

or

$$
\chi_{r+2}=D_{\omega}^{-1}\left(\tilde{h}_{r+2}^{(r)}\right)
$$

where $\tilde{h}_{r+2}^{(r)}=h_{r+2}^{(r)}-Z_{r+2}$ denotes the fluctuating part of $h_{r+2}^{(r)}$. But $\tilde{h}_{r+2}^{(r)}=\tilde{h}_{r+2}^{(r-2)}+L_{\chi_{r}} Z_{4}$. Therefore we get

$$
\chi_{r+2}=D_{\omega}^{-1}\left(\tilde{h}_{r+2}^{(r-2)}+L_{\chi_{r}} Z_{4}\right)
$$

The last equation clearly shows that all the Fourier modes of $\chi_{r}$ propagate unaltered into $\chi_{r+2}$, while new Fourier modes (of order $r+2$ ) are introduced only by the Hamiltonian at order $r+2$.

In the same way, studying the interaction of $\chi_{r}$ with any term of the normal form $Z_{s}$, where $s \leqslant r-1$, by means of the Lie triangle, we find that the Fourier modes of $\chi_{r}$ propagate according to the scheme

$$
\chi_{r} \rightarrow L_{\chi_{r}} Z_{s} \rightarrow \chi_{r+s-2} \rightarrow L_{\chi_{r+s-2}} Z_{s} \rightarrow \chi_{r+2 s-4} \rightarrow L_{\chi_{r+2 s-4}} Z_{s} \rightarrow \cdots
$$

In particular, by combining the equations above in the form

$$
L_{\chi_{r}} Z_{s}=\left\{D_{\omega}^{-1} \tilde{h}_{r}^{(r-2)}, Z_{s}\right\}
$$


and setting $s=4$, we find

$$
\chi_{r+4}=D_{\omega}^{-1} \tilde{h}_{r+4}^{(r)}+D_{\omega}^{-1}\left\{D_{\omega}^{-1} \tilde{h}_{r+2}^{(r-2)}, Z_{4}\right\}+D_{\omega}^{-1}\left\{D_{\omega}^{-1}\left\{D_{\omega}^{-1} \tilde{h}_{r}^{(r-4)}, Z_{4}\right\}, Z_{4}\right\}+\cdots
$$

Thus, if we denote by $F_{r}$ the sum of Fourier modes of order $r$, we have from equation (32)

$$
\chi_{r+4} \sim \frac{1}{a_{r+4}} F_{r+4}+\frac{O(r+2)}{a_{r+2}^{2}} F_{r+2}+\frac{O^{2}(r)}{a_{r}^{3}} F_{r}+\cdots
$$

where $a_{r}$ denotes the smallest divisor of order $r$ as defined by (5). The last equation clearly shows that the Fourier modes $F_{r+4}, F_{r+2}, F_{r}, \ldots$, grow independently. Each of them grows geometrically with a ratio

$$
\lambda_{r}=\frac{O(r)}{a_{r}} .
$$

The numerator of equation (34) is a quantity of order $O(r)$. The factor $\lambda_{r}$ can be defined more precisely in the following way. Let $J_{1}^{m_{1} / 2} J_{2}^{m_{2} / 2}$ be any term of $Z_{4}$, i.e. $m_{1}+m_{2}=4$. Let also $J_{1}^{s_{1} / 2} J_{2}^{s_{2} / 2} \mathrm{e}^{\mathrm{i} k_{1} \phi_{1}+\mathrm{i} k_{2} \phi_{2}}$ be any term of $\chi_{r}, s_{1}+s_{2}=r,\left|k_{1}\right|+\left|k_{2}\right| \leqslant r$ and of the same parity as $r$. Then the Poisson bracket for these two particular terms gives

$$
\begin{aligned}
\left\{J_{1}^{S_{1} / 2} J_{2}^{S_{2} / 2} \mathrm{e}^{\mathrm{i} k_{1} \phi_{1}+\mathrm{i} k_{2} \phi_{2}}, J_{1}^{m_{1} / 2} J_{2}^{m_{2} / 2}\right\} & \\
= & \frac{\mathrm{i}}{2}\left(k_{1} m_{1} J_{1}^{\frac{s_{1}+m_{1}-2}{2}} J_{2}^{\frac{s_{2}+m_{2}}{2}}+k_{2} m_{2} J_{1}^{\frac{s_{1}+m_{1}}{2}} J_{2}^{\frac{s_{2}+m_{2}-2}{2}}\right) \mathrm{e}^{\mathrm{i} k_{1} \phi_{1}+\mathrm{i} k_{2} \phi_{2}} .
\end{aligned}
$$

Thus we have, in compact notation,

$$
\left\|D_{\omega}^{-1}\left\{J^{\frac{s}{2}} \mathrm{e}^{\mathrm{i} k \cdot \phi}, J^{\frac{m}{2}}\right\}\right\| \leqslant \frac{|k \| m|}{2|k \cdot \omega|} \leqslant \frac{2|k|}{|k \cdot \omega|}
$$

where $|k|=\sqrt{k_{1}^{2}+k_{2}^{2}}$. In view of equation (29), it follows that the term $J_{1}^{s_{1} / 2} J_{2}^{s_{2} / 2} \mathrm{e}^{\mathrm{i} k_{1} \phi_{1}+\mathrm{i} k_{2} \phi_{2}}$ propagates in the generating function $\chi_{r+2}$ with a size larger with respect to its size in $\chi_{r}$ by a ratio

$$
\lambda_{k}=\frac{2|k| A}{|k \cdot \omega|}
$$

where $A$ is the supremum norm of $Z_{4}$.

3.2.3. Dominant Fourier modes and growth of the size of the generating function. If we set $\lambda_{r}=\max \left\{\frac{2|k| A}{|k \cdot \omega|},\left|k_{1}\right|+\left|k_{2}\right| \leqslant r\right.$, where $\left.\left(\left|k_{1}\right|+\left|k_{2}\right|\right) \bmod 2=r \bmod 2\right\}$

then, in view of equation (32), we have

$$
\left\|\chi_{r}\right\| \sim \sum_{\kappa=0,2, \ldots, r-6}\left\|D_{\omega}^{-1}\left(D_{\omega}^{-1} L_{Z_{4}}\right)^{\kappa / 2} \tilde{h}_{r-\kappa}^{(r-\kappa-4)}\right\| .
$$

But, due to equation (36), there is no term in $\left(D_{\omega}^{-1} L_{Z_{4}}\right)^{v} \tilde{h}_{r}^{(r-4)}$ that can grow faster than $\left(\lambda_{r}\right)^{v}$. Thus, after $v$ repetitions, the size of the $F_{r}$ terms $\left(D_{\omega}^{-1} L_{Z_{4}}\right)^{v} \tilde{h}_{r}^{(r-4)}$ in $\chi_{r+2 v}$ is bounded by

$$
\left\|F_{r, r+2 v}\right\| \leqslant\left(\lambda_{r}\right)^{v}\left\|F_{r, r}\right\| \leqslant\left(\lambda_{r}\right)^{v}\left\|D_{\omega}^{-1} \tilde{h}_{r}^{(r-4)}\right\|
$$

where $F_{i, j}$ denotes the $F_{i}$ terms of $\chi_{j}$.

Thus the overall picture of the growth of the size of the generating function is the following. In the generating function up to order $r$ there are terms corresponding to Fourier modes $r, r-2, r-4, \ldots$ In the next iterations, $\chi_{r+2}, \chi_{r+4}, \ldots$, the size of each of these modes grows independently with a geometrical factor $\lambda_{r}, \lambda_{r-2}, \lambda_{r-4}, \ldots$, respectively. The overall size of $\chi_{r}$ is determined by the size of the largest growing Fourier mode. The initial 
size of the Fourier modes of order $r$ is equal to their size in $\tilde{h}_{r}^{(r-4)}$. As $r$ increases, new factors $\lambda$, larger than the previous factors, are expected to appear at those specific orders where new diophantine divisors appear. These orders are specified by the continuous fraction expansion of the frequency ratio, as explained in sections 2.1 and 2.2. When a new diophantine divisor appears at some order, its corresponding Fourier mode grows with a factor larger than the factor of the previous modes. These consecutive appearances of new factors by abrupt steps produce a growth of the size of the series that looks piecewise geometrical. Namely, this is a growth with an apparently constant factor for many orders, which gives the impression that there is a constant, non-zero, radius of convergence. However, at some subsequent order the factor increases abruptly and the radius of convergence decreases abruptly. This picture is similar to that found by Servizi et al (1983) in the case of two-dimensional symplectic mappings of the plane.

If $a_{3}$ denotes the smallest divisor of order 3 , then $A \sim\left\|Z_{4}\right\| \simeq\left\|H_{3}\right\|^{2} / a_{3}$. Thus, for a given Hamiltonian $\mathrm{H}_{3}$, equation (38) implies that $\lambda_{r}$ depends essentially only on the number theoretical properties of the frequencies $\omega \equiv\left(\omega_{1}, \omega_{2}\right)$. In particular, the maximum of $|k| /|k \cdot \omega|$ is expected to occur for the minimum divisor $|k \cdot \omega|$ because, for this divisor, $|k|$ is still relatively large and it is divided by a small number. On the other hand, away from the minimum divisor, both $|k|$ and $|k \cdot \omega|$ are large and their ratio, as $|k| \rightarrow \infty$, tends to a constant $\simeq 1 /|\omega|$. At any rate, $\lambda_{r}$ can be found immediately once $\omega$ is given. Figure $5(a)$ shows the factor $\lambda_{r}$ as a function of $r$ for the case $\omega_{2} / \omega_{1}=(\sqrt{5}-1) / 2$, for $r$ even (left panel) and for $r$ odd (right panel). Figure 5(b) shows the same function for the case $\omega_{2} / \omega_{1}=12^{1 / 4}-1$. In both cases, the function $\lambda_{r}$ increases by abrupt steps. A comparison of figure $5(a)$ with figure 1 shows that the steps occur at the orders $r$ where the value of the minimum divisor $a_{r}=|k \cdot \omega|$ changes abruptly. Some secondary steps may also appear at orders corresponding to the appearance of low-order multiples of a diophantine divisor. The same is true in the case of figure $5(b)$, as seen from a comparison with figure 4(a).

In figure 5(a) the plateaux appear in quite regular intervals on a logarithmic scale so that an average power law fit can be found. This is shown as a straight line in figure 5(a). The best fit power law is

$$
\lambda_{r} \simeq 0.036 r^{1.67} \quad r \text { even, } \quad \lambda_{r} \simeq 0.020 r^{2.03} \quad r \text { odd. }
$$

The power exponent found for odd orders is about equal to $1+\tau=2$ ( $\tau=1$ in this case), while the exponent found for even orders differs from $1+\tau$ by about 0.3 . We expect to obtain approximately the value $1+\tau$ of the exponent because each new divisor $k \cdot \omega$ is $O\left(r^{\tau}\right)$, while the numerator $|k|$ of $\lambda_{r}$ given by equation (38) is also $O(r)$. However, it should be emphasized that this average power law applies only because the successive steps are at quite regular intervals, a fact due to the nature of the golden mean number. On the other hand, in the case of the less noble number $12^{1 / 4}-1$ (figure $5(b)$ ) the steps and subsequent plateaux appear at irregular intervals of values of $r$, and one must go to orders much higher than 120 in order to obtain a good number of plateaux that allow us to draw a meaningful power law.

\subsection{Accumulation of small divisors in the direct method}

3.3.1. Old estimates. An approximate treatment of the problem of the accumulation of small divisors in the direct method is made in Giorgilli (1988). A law of the form

$$
\left\|R_{r}\right\| \sim\left\|\Phi_{r}\right\| \sim r !^{1+\tau}
$$

was found. We summarize first the main steps that lead to an estimate of the form (42). 
Consider the recursion relation

$$
D_{\omega} \Phi_{r+1}=-\left[\Phi_{r}, H_{3}\right]
$$

Any term of $\Phi_{r}$ is written as

$$
J_{1}^{s_{1} / 2} J_{2}^{s_{2} / 2} \exp \left(\mathrm{i}\left(k_{1} \phi_{1}+k_{2} \phi_{2}\right)\right)
$$

with $s_{1}+s_{2}=r,\left|k_{1}\right|+\left|k_{2}\right| \leqslant r$ and $\left|k_{1}\right|+\left|k_{2}\right|$ is of the same parity as $r$. Similarly, any term of $H_{3}$ is written as

$$
J_{1}^{m_{1} / 2} J_{2}^{m_{2} / 2} \exp \left(\mathrm{i}\left(n_{1} \phi_{1}+n_{2} \phi_{2}\right)\right)
$$

with $m_{1}+m_{2}=3,\left|n_{1}\right|+\left|n_{2}\right|=1$ or 3 . The Poisson bracket of the two terms gives

$$
\begin{gathered}
\frac{\mathrm{i}}{2}\left[\left(k_{1} m_{1}-s_{1} n_{1}\right) J_{1}^{\left(s_{1}+m_{1}-2\right) / 2} J_{2}^{\left(s_{2}+m_{2}\right) / 2}+\left(k_{2} m_{2}-s_{2} n_{2}\right) J_{1}^{\left(s_{1}+m_{1}\right) / 2} J_{2}^{\left(s_{2}+m_{2}-2\right) / 2}\right] \\
\times \exp \left(\mathrm{i}\left(k_{1}+n_{1}\right) \phi_{1}+\mathrm{i}\left(n_{2}+k_{2}\right) \phi_{2}\right) .
\end{gathered}
$$

So the new term in the integral reads

$$
\begin{aligned}
\frac{\mathrm{i}}{2} D_{\omega}^{-1}\left\{\left[\left(k_{1} m_{1}\right.\right.\right. & \left.\left.-s_{1} n_{1}\right) J_{1}^{\left(s_{1}+m_{1}-2\right) / 2} J_{2}^{\left(s_{2}+m_{2}\right) / 2}+\left(k_{2} m_{2}-s_{2} n_{2}\right) J_{1}^{\left(s_{1}+m_{1}\right) / 2} J_{2}^{\left(s_{2}+m_{2}-2\right) / 2}\right] \\
& \left.\times \exp \left(\mathrm{i}\left(k_{1}+n_{1}\right) \phi_{1}+\mathrm{i}\left(n_{2}+k_{2}\right) \phi_{2}\right)\right\} \\
= & \frac{\frac{1}{2}\left[\left(k_{1} m_{1}-s_{1} n_{1}\right) J_{1}^{\left(s_{1}+m_{1}-2\right) / 2} J_{2}^{\left(s_{2}+m_{2}\right) / 2}+\left(k_{2} m_{2}-s_{2} n_{2}\right) J_{1}^{\left(s_{1}+m_{1}\right) / 2} J_{2}^{\left(s_{2}+m_{2}-2\right) / 2}\right]}{\left(k_{1}+n_{1}\right) \omega_{1}+\left(k_{2}+n_{2}\right) \omega_{2}} \\
& \times \exp \left(\mathrm{i}\left(k_{1}+n_{1}\right) \phi_{1}+\mathrm{i}\left(n_{2}+k_{2}\right) \phi_{2}\right) .
\end{aligned}
$$

The norm of the numerator in equation (45) is $O(r)$ because of the $s_{1} n_{1}$ and $s_{2} n_{2}$ terms, where $s_{1}+s_{2}=r$. On the other hand, the smallest denominator $(k+n) \cdot \omega$ is given by the diophantine condition $(k+n) \cdot \omega \sim a_{r+1}=O\left(1 /(r+1)^{\tau}\right)$. Therefore

$$
\left\|\Phi_{r+1}\right\| \sim \frac{O(r+1)}{a_{r+1}}\left\|\Phi_{r}\right\| \sim O\left((r+1)^{\tau+1}\right)\left\|\Phi_{r}\right\| .
$$

Going backwards, after $r-2$ iterations we find

$$
\left\|\Phi_{r+1}\right\| \sim \frac{O(r+1) O(r) \cdots O(3)}{a_{r+1} a_{r} \cdots a_{3}} \sim O\left((r+1) !^{\tau+1}\right) .
$$

3.3.2. Divisor paths and repetitions in the direct method. By the above analysis, there appears to be an accumulation of divisors in the terms of a formal integral series in the form of products $a_{r+1} a_{r} \cdots a_{3}$. However, this is a simplification. We show now that there cannot be any term in $\Phi_{r+1}$ which contains a product of divisors of the form $a_{r+1} a_{r} \cdots a_{3}$. This is due to the following remark. The term (44) appearing in the above construction is itself produced by the recursion relation (43) in the previous step, namely

$$
D_{\omega} \Phi_{r}=-\left[\Phi_{r-1}, H_{3}\right] \text {. }
$$

Therefore this term is produced by dividing a Fourier term $\exp (\mathrm{i} k \cdot \phi)$ in $\left[\Phi_{r-1}, H_{3}\right]$ by $k \cdot \omega$. Similarly, the term $\exp (\mathrm{i}(k+n) \cdot \phi)$ appearing in equation (45) is divided by $k^{\prime} \cdot \omega=k \cdot \omega+$ $n \cdot \omega$, where $n \equiv\left(n_{1}, n_{2}\right)$ is some fixed integer vector corresponding to a particular Fourier mode in $H_{3}$ (in its most general form, $H_{3}$ contains all terms with $\left|n_{1}\right|+\left|n_{2}\right|=1$ or 3 ). Therefore, the two successive divisors appearing in the term $\exp (\mathrm{i}(n+k) \cdot \phi)$ of $\Phi_{r+1}$ are linked by

$$
k^{\prime} \cdot \omega=k \cdot \omega+A_{j}
$$


where $A_{j}=n_{j} \cdot \omega, j=1, \ldots, M$ corresponds to any of the $M$ different Fourier modes $\exp \left(\mathrm{in}_{j} \cdot \phi\right)$ that appear in $H_{3}$.

Let now $A=\min \left\{\left|A_{j}\right|\right\}$. If $k \cdot \omega$ is diophantine then we have $|k \cdot \omega| \ll A$. But then, equation (49) implies that $\left|k^{\prime} \omega\right|=O(A)$, i.e. $k^{\prime} \cdot \omega$ cannot be diophantine. Similarly, if $k^{\prime} \cdot \omega$ is diophantine, $|k \cdot \omega|=O(A)$, i.e. $k \cdot \omega$ is not diophantine. Thus, two successive divisors in the sequence $a_{3}, a_{4}, \ldots, a_{r+1}$ cannot both be diophantine.

On the other hand, if for some $j_{0} \geqslant 3$ the divisor $a_{j 0}=k \cdot \omega$ is diophantine, then there are terms in $\Phi_{r+1}$ such that every second divisor $a_{j 0+2}, a_{j 0+4}, \ldots$ in the sequence $a_{j}$ for these particular terms, for $j>j_{0}$, is equal to $a_{j 0}$. To see this, we note that equation (49) implies that the paths of successive divisors

$$
k \cdot \omega \rightarrow k^{\prime} \cdot \omega \rightarrow k^{\prime \prime} \cdot \omega \rightarrow \cdots
$$

can be arranged in a tree graph starting with a single node $\left(a_{j 0}=k \cdot \omega\right)$ with $M$ branches. As the recursion relation (43) is iterated, in the second step the endpoint of each branch is a starting node for $M$ new branches. In particular, if we take the Poisson bracket of the initial term $\exp (\mathrm{i} k \cdot \omega)$ of $\Phi_{j 0}$ alternatively with the $H_{3}$ terms $\exp (\mathrm{i} n \cdot \omega), \exp (-\mathrm{i} n \cdot \omega)$, $\exp (\mathrm{i} n \cdot \omega), \ldots$, we create terms corresponding to Fourier modes $\exp (\mathrm{i}(k+n) \cdot \omega), \exp (\mathrm{i} k \cdot \omega)$, $\exp (\mathrm{i}(k+n) \cdot \omega), \ldots$, in subsequent orders of $\Phi$ which are divided by the sequence of divisors

$$
k \cdot \omega \rightarrow(k+n) \cdot \omega \rightarrow k \cdot \omega \rightarrow \cdots .
$$

Such a sequence of Fourier terms, and of their respective divisors, will be called a repetition path.

Repetition paths are important because they introduce the same diophantine divisor $k \cdot \omega=a_{j 0}$ in every second step. The growth of the size of the series terms produced by repetition paths is piecewise geometrical. This can be seen by using the direct method as above, but the calculation is simplified if we implement the direct method not in the Hamiltonian $H=H_{2}+H_{3}$, but in the Hamiltonian $H^{(1)}$ after just one normalization step, namely $H^{(1)}=\exp \left(L_{\chi_{3}}\right) H$. The new Hamiltonian reads

$$
H^{(1)}=Z_{2}+h_{4}^{(1)}+h_{5}^{(1)}+\cdots
$$

where $h_{s}^{(1)}$ is of order $s / 2$ in the actions. The recursion relation for the direct method reads

$$
D_{\omega} \Phi_{r+2}=-\left[\Phi_{r}, h_{4}^{(1)}\right]+\cdots .
$$

The gain from the above normalization is that one iteration of equation (51) corresponds essentially to two iterations of equation (43), i.e. of the recursion relation before the normalization.

Taking now again the Poisson bracket of a term of $\Phi_{r}$, written as (44) with $s_{1}+s_{2}=$ $r,\left|k_{1}\right|+\left|k_{2}\right| \leqslant r$ and of the same parity as $r$, with the term

$$
J_{1}^{m_{1} / 2} J_{2}^{m_{2} / 2} \exp \left(\mathrm{i}\left(n_{1} \phi_{1}+n_{2} \phi_{2}\right)\right)
$$

of $h_{4}^{(1)}$, with $m_{1}+m_{2}=4,\left|n_{1}\right|+\left|n_{2}\right| \leqslant 4$, yields two terms in $R_{r+2}$ given by

$$
\begin{gathered}
\frac{\mathrm{i}}{2}\left[\left(k_{1} m_{1}-s_{1} n_{1}\right) J_{1}^{\left(s_{1}+m_{1}-2\right) / 2} J_{2}^{\left(s_{2}+m_{2}\right) / 2}+\left(k_{2} m_{2}-s_{2} n_{2}\right) J_{1}^{\left(s_{1}+m_{1}\right) / 2} J_{2}^{\left(s_{2}+m_{2}-2\right) / 2}\right] \\
\times \exp \left(\mathrm{i}\left(k_{1}+n_{1}\right) \phi_{1}+\mathrm{i}\left(k_{2}+n_{2}\right) \phi_{2}\right) .
\end{gathered}
$$

The corresponding new terms in $\Phi_{r+2}$ are

$$
\begin{aligned}
\frac{\mathrm{i}}{2} D_{\omega}^{-1}\left\{\left[\left(k_{1} m_{1}\right.\right.\right. & \left.\left.-s_{1} n_{1}\right) J_{1}^{\left(s_{1}+m_{1}-2\right) / 2} J_{2}^{\left(s_{2}+m_{2}\right) / 2}+\left(k_{2} m_{2}-s_{2} n_{2}\right) J_{1}^{\left(s_{1}+m_{1}\right) / 2} J_{2}^{\left(s_{2}+m_{2}-2\right) / 2}\right] \\
& \left.\times \exp \left(\mathrm{i}\left(k_{1}+n_{1}\right) \phi_{1}+\mathrm{i}\left(n_{2}+k_{2}\right) \phi_{2}\right)\right\}
\end{aligned}
$$




$$
\begin{aligned}
= & \frac{\frac{1}{2}\left[\left(k_{1} m_{1}-s_{1} n_{1}\right) J_{1}^{\left(s_{1}+m_{1}-2\right) / 2} J_{2}^{\left(s_{2}+m_{2}\right) / 2}+\left(k_{2} m_{2}-s_{2} n_{2}\right) J_{1}^{\left(s_{1}+m_{1}\right) / 2} J_{2}^{\left(s_{2}+m_{2}-2\right) / 2}\right]}{\left(k_{1}+n_{1}\right) \omega_{1}+\left(k_{2}+n_{2}\right) \omega_{2}} \\
& \times \exp \left(\mathrm{i}\left(k_{1}+n_{1}\right) \phi_{1}+\mathrm{i}\left(n_{2}+k_{2}\right) \phi_{2}\right) .
\end{aligned}
$$

The norms of the terms in the numerator of (52) are $\left|k_{1} m_{1}-s_{1} n_{1}\right|$ and $\left|k_{2} m_{2}-s_{2} n_{2}\right|$. If we add and subtract $n_{1} m_{1}$ inside the first absolute value and $n_{2} m_{2}$ inside the second absolute value, we find that the numerator is smaller than $|k+n| \cdot m+(s+m) \cdot|n|$, where $m$ and $s$ are positive. Thus we find

$$
\begin{gathered}
\left\|\frac{\frac{1}{2}\left[\left(k_{1} m_{1}-s_{1} n_{1}\right) J_{1}^{\left(s_{1}+m_{1}-2\right) / 2} J_{2}^{\left(s_{2}+m_{2}\right) / 2}+\left(k_{2} m_{2}-s_{2} n_{2}\right) J_{1}^{\left(s_{1}+m_{1}\right) / 2} J_{2}^{\left(s_{2}+m_{2}-2\right) / 2}\right]}{\left(k_{1}+n_{1}\right) \omega_{1}+\left(k_{2}+n_{2}\right) \omega_{2}}\right\| \\
\leqslant \frac{1}{2} \frac{|k+n| \cdot m+|s+m| \cdot|n|}{|k+n| \cdot \omega} .
\end{gathered}
$$

Repetition paths are obtained simply by observing that $h_{4}^{(1)}$ contains in general a part in normal form $Z_{4}=\left\langle h_{4}^{(1)}\right\rangle$, which corresponds to the Fourier mode $n=0$. But then, according to equation (52), the Poisson bracket $\left[\Phi_{r}, Z_{4}\right]$ creates just the repetition of the Fourier mode $\exp \left(\mathrm{i} k^{\prime} \cdot \omega\right)=\exp (\mathrm{i} k \cdot \omega)$ (since $\left.n=0\right)$ iteratively in $\Phi_{r}, \Phi_{r+2}, \Phi_{r+4}, \ldots$ Furthermore, according to equation (53) the growth of the size of this Fourier mode is bounded by $\frac{1}{2}\left|\frac{|k| m}{k \cdot \omega}\right| \leqslant \frac{2|k|}{|k \cdot \omega|}$. Therefore, we recover the same result as by the normal form method, namely that repetition paths introduce terms corresponding to repeated Fourier modes, each growing independently from each other with the ratio $\lambda_{r}$ given by equation (38).

3.3.3. Contributions by non-repetition paths. The normal form terms $n=0$ will be said to produce repetition paths of period 1, since every successive term produced iteratively corresponds to the same Fourier mode, i.e. this mode is repeated at every iteration.

We consider now the growth of the size of terms produced by paths corresponding to $n \neq 0$ in equations (52) and (53). Let $r_{0}$ be the order at which a new diophantine divisor appears. We consider the growth, for $r \geqslant r_{0}$, of terms produced either by non-repetition paths or by repetition paths of period larger than 1 . Among these terms, the fastest growing are those corresponding to repetition paths of period 2. For these terms we have $|k+n| \cdot m+$ $(s+m) \cdot|n|=O(r)$, because $s_{1}+s_{2}=r$, and $|m n| \ll|s n|$ for $r$ large. On the other hand, a divisor $\sim r_{0}^{-\tau}$ appears in every second iteration. Thus, after $v$ iterations beyond $r=r_{0}$, the size of the respective terms has grown as $O\left(r_{0}\left(r_{0}+2\right) \cdots\left(r_{0}+2 \nu\right) r_{0}^{\nu \tau / 2}\right)$. This is to be compared with the size of the terms produced by repetition paths of period 1 , which is $\left(\lambda_{r_{0}}\right)^{\nu}=O\left(r_{0}^{\nu(\tau+1)}\right)$. Clearly, $r_{0}^{\nu(\tau+1)} \gg r_{0}\left(r_{0}+2\right) \cdots\left(r_{0}+2 \nu\right) r_{0}^{\nu \tau / 2}$ as long as

$$
v<\frac{r_{0}^{1+\frac{\tau}{2}}-r_{0}}{2} .
$$

In the 2 DOF case $\tau=1$ so that repetition paths of period 2 become of equal size to repetition paths of period 1 after $2 v \sim r_{0}^{1.5}$ iterations. But in the meantime, many more new diophantine divisors appear, as $r$ increases, which create terms growing much faster than the terms with divisor $a_{r_{0}}$ appearing in repetition paths of any period. For example, in the case of the golden mean, the diophantine divisor $a_{34}=13 \omega_{1}-21 \omega_{2}$ appears at order $r_{0}=34$. Thus, repetition paths of period 2 with this particular divisor produce terms of size comparable to the size of terms with the same divisor produced by period 1 repetitions after $2 v \simeq 200$ iterations, i.e. at the order $r=2 v+34=234$. But there are 3 new diophantine divisors after $a_{34}$ which appear up to order 234, and these divisors produce terms which, at $r=234$, are in any case far more important than the terms produced by the repetition, of any period, of the $F_{34}$ mode. 
We conclude that non-repetition paths, or repetition paths of period higher than 1 can be safely ignored. Thus the direct method gives essentially the same result as the normal form method, namely that the growth of the size of the series terms is piecewise geometrical, with ratio $\lambda_{r}$ changing abruptly at particular values of $r$ where new diophantine divisors appear.

\subsection{Delays and inversions}

3.4.1. Delays. Let $r_{0}$ be the order at which a new diophantine divisor $a_{r 0}=k \cdot \omega$ appears, and $r_{0}^{\prime}>r_{0}$ be the order at which the next new diophantine divisor $a_{r 0^{\prime}}=k^{\prime} \omega$ appears. We assume $r_{0}$ and $r_{0}^{\prime}$ to have the same parity. The size of the Fourier mode $\exp (\mathrm{i} k \cdot \phi)$ grows with a geometrical factor $\lambda_{r 0} \sim r_{0}^{\tau+1}$, while the size of the Fourier mode $\exp \left(\mathrm{i} k^{\prime} \phi\right)$ grows with a geometrical factor $\lambda_{r 0^{\prime}} \sim r_{0}^{\prime \tau+1}>\lambda_{r 0}$. The recursion relation (43) implies that there are at least $\left(r_{0}^{\prime}-r_{0}\right) / 3$ steps needed to reach the mode $\exp \left(\mathrm{i} k^{\prime} \phi\right)$, at order $r_{0}^{\prime}$, starting from the mode $\exp (\mathrm{i} k \cdot \phi)$, through a non-repetition path. On the other hand, it is possible to start from the mode $\exp (\mathrm{i} k \cdot \phi)$ and remain in the same mode up to the order $r_{0}^{\prime}$ via a repetition path. In the latter path, the mode $\exp (\mathrm{i} k \cdot \phi)$ gains a factor $\lambda_{r 0}$ at every second step. Therefore when the new mode $\exp \left(\mathrm{i} k^{\prime} \phi\right)$ appears, at order $r_{0}^{\prime}$, the old mode $\exp (\mathrm{i} k \cdot \phi)$ has a size larger than the size of the new mode by a factor $\sim \lambda_{r 0}^{\left(r_{0}^{\prime}-r_{0}\right) / 6}$. From there on, the new mode grows faster than the old mode since $\lambda_{r_{0}^{\prime}}>\lambda_{r_{0}}$. Therefore, the size of the new mode will become larger than the size of the old mode at some order $r>r_{0}^{\prime}$, i.e. the new mode becomes dominant at an order larger than the order of its appearance. We call this phenomenon a 'delay'. Quantitatively, the delay is equal to the difference $r-r_{0}^{\prime}$.

The delay can be estimated as follows. Let $v$ be the number of double steps needed after $r_{0}^{\prime}$ so that the mode $\exp \left(\mathrm{i} k^{\prime} \phi\right)$ acquires a size equal to the size of $\exp (\mathrm{i} k \cdot \phi)$. Then we have

$$
\left(r_{0}^{\tau+1}\right)^{\frac{r_{0}^{\prime}-r_{0}}{6}+v} \simeq\left(r_{0}^{\prime \tau+1}\right)^{v}
$$

which yields the estimate

$$
2 v \simeq \frac{\left(r_{0}^{\prime}-r_{0}\right) \log r_{0}}{3 \log \left(r_{0}^{\prime} / r_{0}\right)} .
$$

The Fourier mode $\exp \left(\mathrm{i} k^{\prime} \phi\right)$ becomes dominant shortly thereafter (for larger $r$ ). For example, the mode $\exp \left(\mathrm{i} k^{\prime} \phi\right)$ exceeds by a factor 10 the mode $\exp (\mathrm{i} k \cdot \phi)$ at order

$$
r \simeq \frac{\left(r_{0}^{\prime}-r_{0}\right) \log r_{0}}{3 \log \left(r_{0}^{\prime} / r_{0}\right)}+\frac{2 \log 10}{(\tau+1) \log \left(r_{0}^{\prime} / r_{0}\right)}
$$

Figure 6 displays the delay phenomenon in the case $\omega_{2} / \omega_{1}=12^{1 / 4}-1$. This figure shows the divisor corresponding to the dominant Fourier mode as calculated with the direct method, as a function of the order $r$. This figure is to be compared with figure 4(a). In this figure we see that the divisor $6 \omega_{1}-7 \omega_{2}$ appears at order 13. However, as seen in figure 6, this divisor of figure $4(a)$ produces a dominant Fourier mode exp $\left( \pm \mathrm{i}\left(6 \phi_{1}-7 \phi_{2}\right)\right)$ only at order $r=23$. This is because there is a delay for this mode to prevail over the mode $\exp \left( \pm \mathrm{i}\left(\phi_{1}-\phi_{2}\right)\right)$, which is the previous most important mode (with divisor $\left.\omega_{1}-\omega_{2}\right)$. If we use the estimate of equation (57) with $r_{0}=2, r_{0}^{\prime}=13$, we find $r=17$ which is not too far from $r=23$. Similarly, the Fourier mode $\exp \left( \pm \mathrm{i}\left(31 \phi_{1}-36 \phi_{2}\right)\right)$ appears at the order 67 , but as figure 6 shows, this mode becomes dominant only at order 89. In this case, equation (57), with $r_{0}=13, r_{0}^{\prime}=67$, gives $r=97$ which is not too far from $r=89$. Thus, despite the approximations used, the estimate (57) does not deviate very much from the values found numerically. 


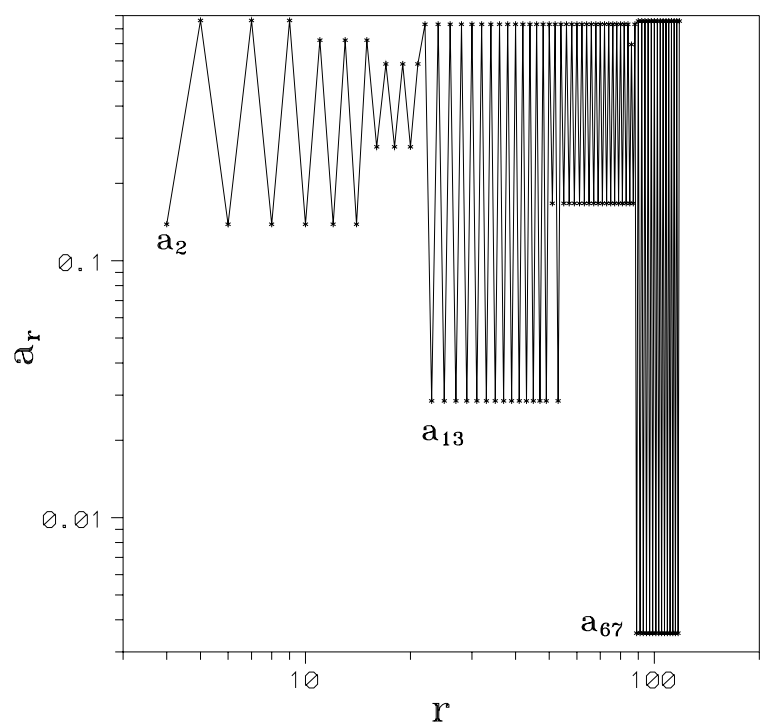

Figure 6. The divisor $a_{r}$ corresponding to the Fourier mode with the biggest size (dominant mode) at order $r$, as a function of $r$. The mode with the divisor $a_{2}$ of figure 4(a) is dominant at $r=4$. The mode with the divisor $a_{13}$ of figure 4(a) becomes dominant at $r=23$. Finally, the mode with the divisor $a_{67}$ of figure 4(a) becomes dominant at $r=89$.

3.4.2. Inversions. Another interesting phenomenon shown in figure 6 is the phenomenon of 'inversion'. Namely, a Fourier mode different from both $\exp \left( \pm \mathrm{i}\left(6 \phi_{1}-7 \phi_{2}\right)\right)$ and $\exp \left( \pm \mathrm{i}\left(31 \phi_{1}-36 \phi_{2}\right)\right)$ becomes dominant for the interval of values $55 \leqslant r \leqslant 88$. This phenomenon appears in the transient interval when new modes with diophantine divisors appear, before these modes become dominant. In the above example, an inversion occurs with the appearance of the modes $\exp \left( \pm \mathrm{i}\left(25 \phi_{1}-29 \phi_{2}\right)\right)$ (at order 54$)$, and $\exp \left( \pm \mathrm{i}\left(31 \phi_{1}-36 \phi_{2}\right)\right)$ (at order 67). In particular, when several new modes appear at nearby orders, there are transient intervals of $r$ in which no mode is clearly dominant. In such intervals even some nonrepetition paths may produce terms with size comparable to the size of the terms produced by repetition paths. Note that the mode $\exp \left( \pm \mathrm{i}\left(25 \phi_{1}-29 \phi_{2}\right)\right)$ never becomes dominant. This is because, as we see in figure 4(a), the order of appearance of this mode (56) is quite close to the order of appearance of the next mode $\exp \left( \pm \mathrm{i}\left(31 \phi_{1}-36 \phi_{2}\right)\right)$, and the latter mode is much more important than the former, because it has a much smaller divisor, i.e. a much larger $\lambda$. Therefore, the delay mechanism produces a smaller delay in the case of the $\exp \left( \pm \mathrm{i}\left(31 \phi_{1}-36 \phi_{2}\right)\right)$ mode than in the case of the $\exp \left( \pm \mathrm{i}\left(25 \phi_{1}-29 \phi_{2}\right)\right)$ mode and the mode $\exp \left( \pm \mathrm{i}\left(31 \phi_{1}-36 \phi_{2}\right)\right)$ becomes dominant before the size of the mode $\exp \left( \pm \mathrm{i}\left(25 \phi_{1}-29 \phi_{2}\right)\right)$ exceeds the size of the previously dominant mode.

\subsection{Explanation of the numerical results}

The phenomena described above can now be compared with numerical results. The piecewise geometrical growth and the inversions are clearly seen if we plot the size of the various Fourier modes $\exp (\mathrm{i}(k \cdot \phi))$ at particular orders versus the corresponding divisor $k \cdot \omega$. This is shown in figure 7 for the case $\omega_{2} / \omega_{1}=12^{1 / 4}-1$. Figure $7(a)$ shows the size of all the Fourier modes in the integral $\Phi_{r}$ with divisors $\left|a_{r}\right| \leqslant 1$ at order $r=14$. The dominant mode is $\exp \left( \pm \mathrm{i}\left(\phi_{1}-\phi_{2}\right)\right)$. This mode produces two nearly equal dominant modes in $\Phi_{15}$, 
(a) $r=14$

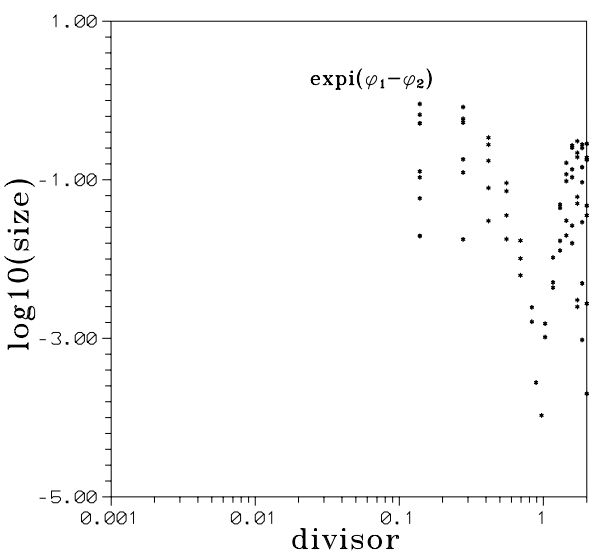

(c) $\mathrm{r}=17$

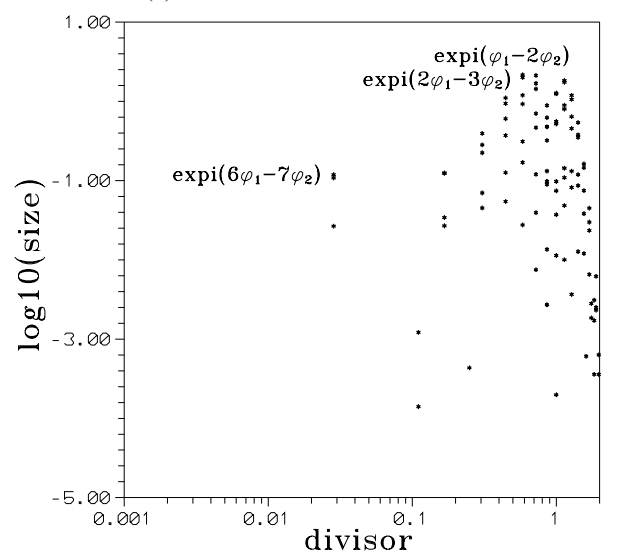

(e) $\mathrm{r}=79$

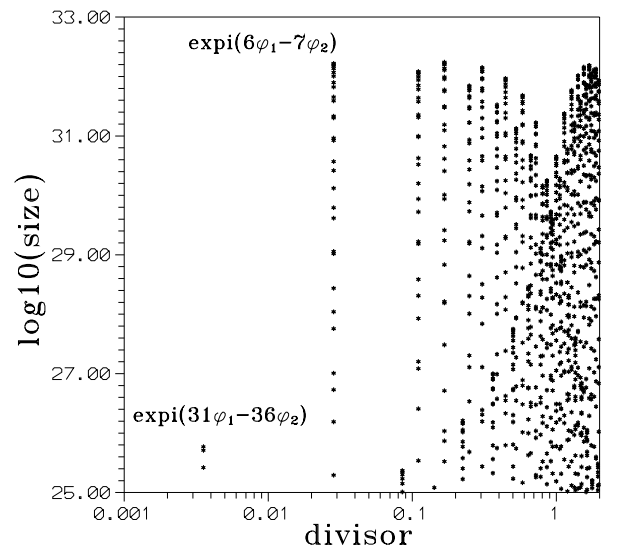

(b) $r=15$

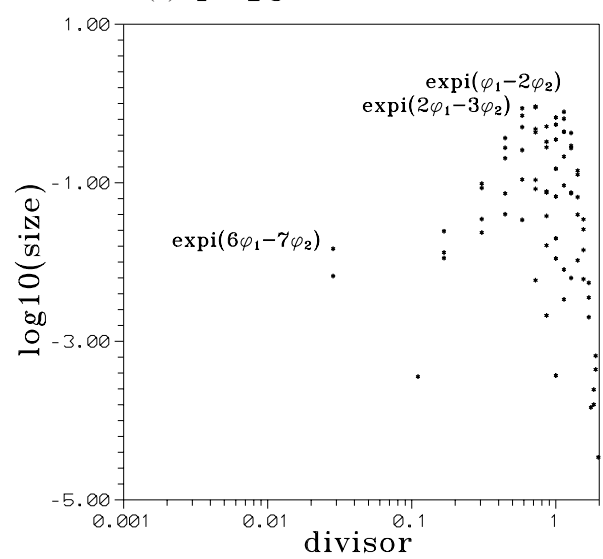

(d) $\mathrm{r}=23$

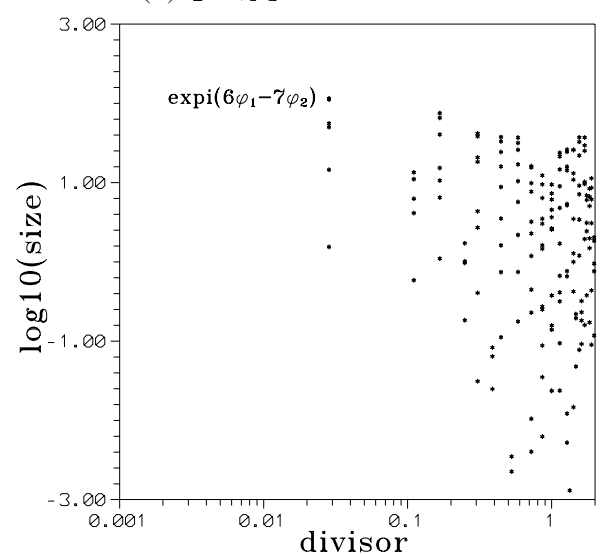

(f) $\mathrm{r}=89$

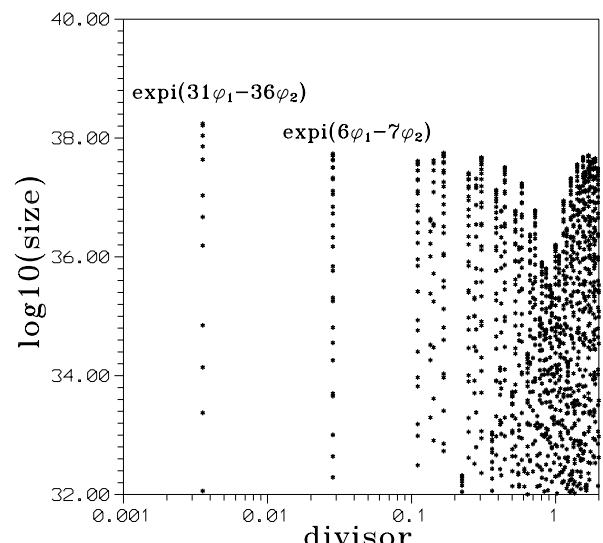

Figure 7. The norm of the various terms of the formal integral at order $r$ as a function of their respective divisor for divisors smaller than $a_{s}=2$. Each point corresponds to one term of the form (44), but there are many terms corresponding to the same Fourier mode and divisor. The Hamiltonian is (3) and the frequencies $\omega_{1}=1, \omega_{2}=12^{1 / 4}-1$. (a) $r=14$, (b) $r=15$, (c) $r=17,(d) r=23,(e) r=79,(f) r=89$. 
(figure 7(b), for $r=15)$, namely $\exp \left( \pm \mathrm{i}\left(2 \phi_{1}-3 \phi_{2}\right)\right)$ and $\exp \left( \pm \mathrm{i}\left(\phi_{1}-2 \phi_{2}\right)\right)$. These terms are produced by the Poisson bracket of $\exp \left(\mathrm{i}\left(\phi_{1}-\phi_{2}\right)\right)$ (the dominant term of $\left.\Phi_{14}\right)$ with the Hamiltonian terms $\exp \left(\mathrm{i}\left(\phi_{1}-2 \phi_{2}\right)\right.$ and $\exp \left(-\mathrm{i} \phi_{2}\right)$, or the Poisson bracket of the complex conjugates $\exp \left(-\mathrm{i}\left(\phi_{1}-\phi_{2}\right)\right)$ with $\exp \left(-\mathrm{i}\left(\phi_{1}-2 \phi_{2}\right)\right)$ and $\exp \left(\mathrm{i} \phi_{2}\right)$ respectively, as explained in section 3.2. Note, however, that there is another mode at $r=15$, namely $\exp \left( \pm \mathrm{i}\left(6 \phi_{1}-7 \phi_{2}\right)\right)$, which corresponds to a smaller divisor than the previous terms, namely $\left|6 \omega_{1}-7 \omega_{2}\right|$. This is the smallest divisor which can appear at $r=15$. The corresponding mode is the fastest growing one. This is shown in figure $7(c)$, at order $r=17$. By comparing the size of the mode $\exp \left( \pm \mathrm{i}\left(6 \phi_{1}-7 \phi_{2}\right)\right)$ at orders $r=15$ and $r=17$ we find a gain of size at $r=17$ by a factor $\lambda \simeq 8.9$. Other modes grow by a smaller factor. Now, the mode $\exp \left( \pm \mathrm{i}\left(6 \phi_{1}-7 \phi_{2}\right)\right)$ has appeared already at order $r=13=6+7$. However, despite the fact that this mode is the fastest growing one, it is not yet dominant at $r=17$ (figure $7(c)$ ) because other modes have a larger size. This mode becomes dominant at $r=23$, as shown in figure $7(d)$, while we find that at subsequent orders it prevails over other modes by orders of magnitude.

Let $S_{r}$ denote the size (norm) of the dominant mode at order $r$. Then at order $r=23$ we have $\log S_{23}=2.05$. The size of the same mode at $r=17$ is $\log S_{17}=-0.85$. Therefore $\log \left(S_{23} / \log S_{17}\right)=2.9$. Since there are three double steps from $r=17$ to $r=23$, assuming geometrical growth, we have $\log \left(S_{23} / \log S_{17}\right)=3 \log \lambda$. Indeed we find $3 \log \lambda=2.85$ which is very close to the value 2.9 found numerically. Thus the growth of the mode is clearly geometrical.

Now, at order $r=67$ there is a newly appearing mode, $\exp \left( \pm \mathrm{i}\left(31 \phi_{1}-36 \phi_{2}\right)\right)$ which corresponds to a smaller divisor $a_{67}=\left|31 \omega_{1}-36 \omega_{2}\right|$ than the divisor $a_{13}$ of the mode $\exp \left( \pm \mathrm{i}\left(6 \phi_{1}-7 \phi_{2}\right)\right)$. Therefore, according to the above considerations, the mode $\exp \left( \pm \mathrm{i}\left(31 \phi_{1}-36 \phi_{2}\right)\right)$, which has initially a very small size, grows at a faster rate than any other mode, including $\exp \left( \pm \mathrm{i}\left(6 \phi_{1}-7 \phi_{2}\right)\right)$ which is dominant at that order. Indeed, we find numerically that the mode $\exp \left( \pm \mathrm{i}\left(31 \phi_{1}-36 \phi_{2}\right)\right)$ grows by a factor $\lambda^{\prime} \simeq 316>8.9=\lambda$. Therefore, after some iterations the mode $\exp \left( \pm \mathrm{i}\left(31 \phi_{1}-36 \phi_{2}\right)\right)$ acquires a significant size. This is shown in figures 7(e) and $(f)$ at $r=79$ and $r=89$. The mode $\exp \left( \pm \mathrm{i}\left(31 \phi_{1}-36 \phi_{2}\right)\right)$ appears to the left of the mode $\exp \left( \pm \mathrm{i}\left(6 \phi_{1}-7 \phi_{2}\right)\right)$ (since it has a smaller divisor). But still the term $\exp \left( \pm \mathrm{i}\left(6 \phi_{1}-7 \phi_{2}\right)\right)$ is larger than $\exp \left( \pm \mathrm{i}\left(31 \phi_{1}-36 \phi_{2}\right)\right)$, for $r=79$. However, at $r=89$ (figure $7(f)$ ), the new mode becomes dominant in its turn. It will remain dominant until new modes, corresponding to smaller divisors, appear at higher orders. This process is repeated ad infinitum.

The ratio $\lambda^{\prime} / \lambda$ is equal to $\simeq 35.5$. This value can be compared with the theoretical estimates given by equation (38). Namely, we have the estimate $\lambda^{\prime} / \lambda=\left(\left|k^{\prime}\right| \omega\right) /\left(|k| \omega^{\prime}\right)$. If we set the appropriate values, namely $|k|=\sqrt{6^{2}+7^{2}}$ and $\left|k^{\prime}\right|=\sqrt{31^{2}+36^{2}}$ we find $\lambda^{\prime} / \lambda=41.9$. Thus the error with respect to the numerically determined value is about $15 \%$.

The fact that the overall growth of the series is piecewise geometrical can be clearly seen if we plot the ratio of the remainders $\left\|R_{r+2}\right\| /\left\|R_{r}\right\|$ as calculated by the direct method. Figure $8(a)$ shows this ratio in the case $\omega_{2} / \omega_{1}=12^{1 / 4}-1$. We can see clearly the formation of plateaux indicating a growth with piecewise constant ratio. The dashed lines correspond to the orders where a particular mode becomes dominant. If we project these particular orders on the diagram $N_{\text {opt }}(\rho)$ (figure $4(b)$, replotted in figure $8(b)$ ), we see that the orders where new abrupt steps occur mark the orders where plateaux are formed in the diagram $N_{\text {opt }}(\rho)$. This is easy to understand. Since the ratio $\left\|R_{r+2}\right\| /\left\|R_{r}\right\|$ is piecewise constant, there are 'pseudoradii of convergence' which are also piecewise constant and equal essentially to the inverse ratio $\left\|R_{r}\right\| /\left\|R_{r+2}\right\|$. Let $\rho_{i}$ and $\rho_{i+1}$ be two successive pseudoradii $\left(\rho_{i+1}<\rho_{i}\right)$. For all $\rho$ with $\rho_{i+1}<\rho<\rho_{i}$ the optimal order of truncation is equal to about the order $r_{i}$ where $\rho_{i}$ appears. 

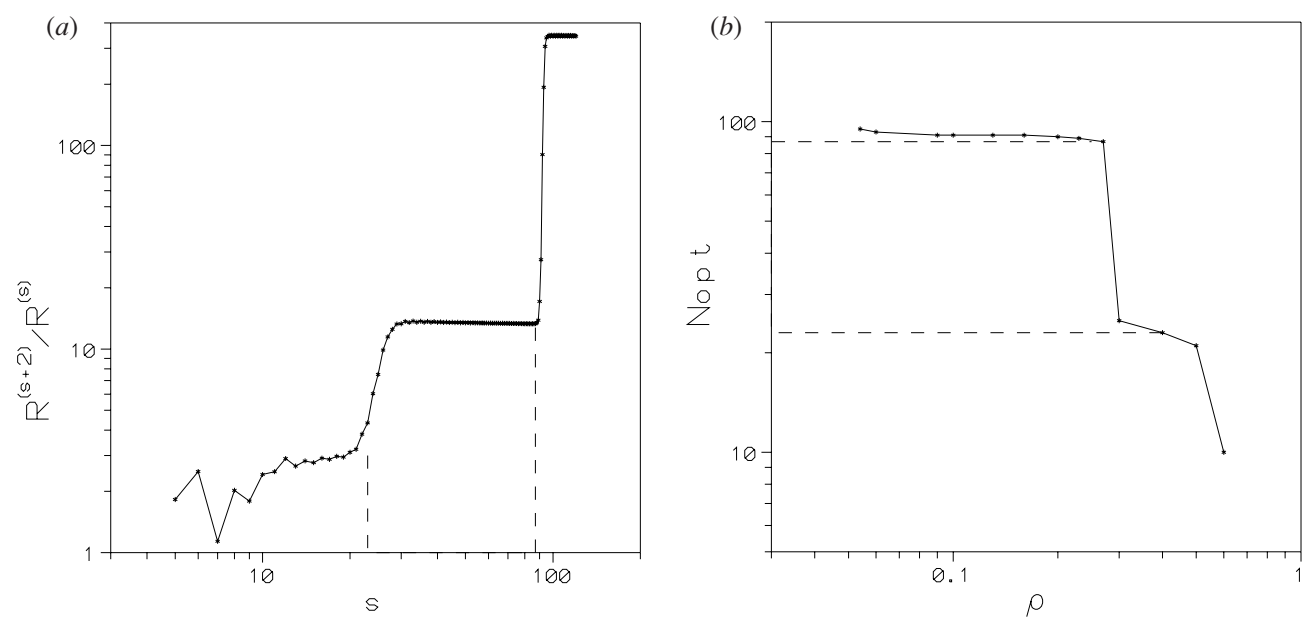

Figure 8. (a) The remainder ratio $\left\|R^{(s+2)}\right\| /\left\|R^{(s)}\right\|$ as a function of $s$ for the formal integral calculated in the Hamiltonian (3) with $\omega_{1}=1, \omega_{2}=12^{1 / 4}-1$. The dashed lines correspond to $s=23$ and $s=89$, i.e. to the orders where the divisors $a_{13}$ and $a_{67}$ become dominant respectively. These orders mark the limits of each plateau in the ratio $\left\|R^{(s+2)}\right\| /\left\|R^{(s)}\right\|$. (b) Figure 4(b) replotted. The dashed lines correspond again to $N_{\mathrm{opt}}=23$ and $N_{\mathrm{opt}}=89$.
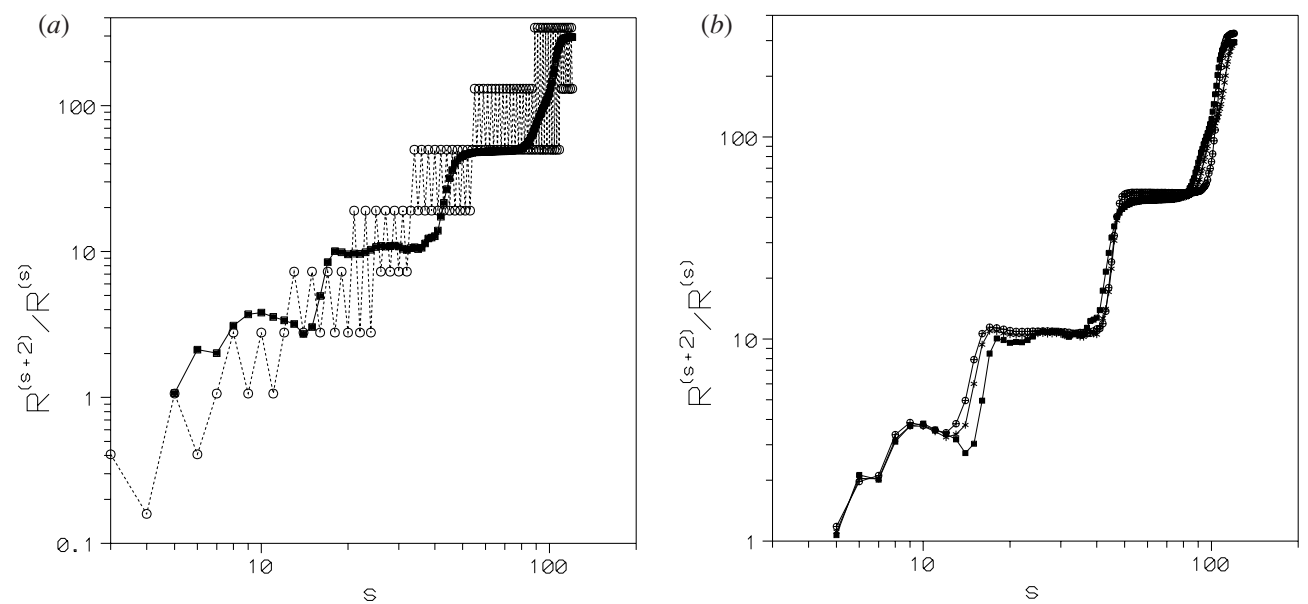

Figure 9. (a) Same as in figure $8(a)$ for the Hamiltonian (3) with $\omega_{1}=1, \omega_{2}=(\sqrt{5}-1) / 2$ (solid line with squares). The dashed line shows the variation of $\lambda_{r}$, as a function of the order $r$, as predicted theoretically (equation (38)). (b) Same as figure 8(a) for the Hamiltonian (60), for the values $\epsilon=0$ (squares), $\epsilon=0.15$ (stars) and $\epsilon=0.30$ (circles).

But the optimal order changes abruptly for $\rho<\rho_{i+1}$ and it moves to $r_{i+1}$. This process is repeated ad infinitum.

Similar phenomena are observed in the case $\omega_{2} / \omega_{1}=(\sqrt{5}-1) / 2$. Figure $9(a)$ shows the ratio $\left\|R_{r+2}\right\| /\left\|R_{r}\right\|$, where again we see the existence of plateaux corresponding to a piecewise constant geometrical growth. These plateaux correspond to the plateaux of figure 5(a). The variations of $\lambda_{r}$ are replotted in figure $9(a)$ as a dashed line going alternatively upwards and downwards. The coincidence with the curve $\left\|R_{r+2}\right\| /\left\|R_{r}\right\|$ is striking, despite the fact that many approximations enter in the theoretical determination $\lambda_{r}$ by the upper bounds 
of the relevant norms. Note also that each plateau in figure $9(a)$ appears with a delay with respect to the orders where new diophantine divisors appear. If we calculate an average power law fitting we find

$$
\frac{\left\|R_{r+2}\right\|}{\left\|R_{r}\right\|}=0.05 r^{1.7}
$$

The power exponent is to be compared with the exponents (equation (41)) found for $\lambda_{r}$. As mentioned earlier, in this case where many plateaux are formed we expect theoretically an average power law $\lambda_{r} \sim r^{1+\tau}$. However, delays are expected to lower somewhat the corresponding exponent for $\left\|R_{r+2}\right\| /\left\|R_{r}\right\|$ versus $r$. Thus we set

$$
\frac{\left\|R_{r+2}\right\|}{\left\|R_{r}\right\|} \sim r^{1+\tau-b}
$$

where $b$ is a 'delay' constant. In general, $b<1$ since delays are only a fraction of the order where new diophantine divisors appear.

In figure $9(b)$ we plot the ratio $\left\|R_{r+2}\right\| /\left\|R_{r}\right\|$ for the formal integral series calculated for more general Hamiltonians of the form

$H \equiv \frac{\omega_{1}}{2}\left(x^{2}+p_{x}^{2}\right)+\frac{\omega_{2}}{2}\left(y^{2}+p_{y}^{2}\right)+0.1\left((1+\epsilon) x^{3}+(1-\epsilon) x^{2} y+x y^{2}+\alpha y^{3}\right)$

where $\alpha$ satisfies the equation

$$
\sqrt{(1+\epsilon)^{2}+(1-\epsilon)^{2}+1+\alpha^{2}}=2
$$

so that the term $\mathrm{H}_{3}$, for all the Hamiltonians of the form (60), has the same size, independent of $\epsilon<1$. For $\epsilon=0$ we find the 'symmetric' Hamiltonian (3) in which all the terms in $\mathrm{H}_{3}$ have equal weights. However, for $\epsilon \neq 0$, the terms have unequal weights and the term $\mathrm{H}_{3}$ no longer has any symmetry with respect to the variables $x$ and $y$. As seen in figure $9(b)$, for three different values $\epsilon=0,0.15$ and 0.30 the behaviour of the function $\left\|R_{r+2}\right\| /\left\|R_{r}\right\|$ is quite similar. Thus, the choice of a symmetric function $H_{3}$ as in equation (3) does not influence the results under consideration. This is due to the fact that equation (38), which determines the growth factor $\lambda_{r}$ at successive orders, does not depend on the symmetry properties of $\mathrm{H}_{3}$, but only on the constant $A$ which depends on the size of $H_{3}$. On the other hand, it should be stressed that one must consider perturbation terms $\mathrm{H}_{3}$ in which all the Fourier modes of order 3 are present. Such terms correspond to the general problem. Otherwise, i.e., if some Fourier modes are absent from $H_{3}$ or if they have relatively small amplitudes with respect to other modes, then the corresponding tree branches in the paths of successive divisors described in section 3 are either absent or ineffective. In this case, the real phenomena regarding the succession of dominant Fourier modes in the series are pushed to higher orders and they are less easily detected numerically.

If we use equation (59) we can estimate now theoretically the law $N_{\text {opt }}(\rho)$. By taking recursively equation (59) we find

$$
\left\|R_{r}\right\| \simeq A^{r / 2} r !^{\frac{1+\tau-b}{2}}
$$

where $A$ is the constant of proportionality in equation (59). Then the norm at distance $\rho$ is given by

$$
\left\|R_{r}\right\|_{\rho} \simeq A^{r / 2} r !^{\frac{1+\tau-b}{2}} \rho^{r} .
$$

Taking logarithms and Stirling's formula we find

$$
\log \left\|R_{r}\right\|_{\rho} \simeq r \log \left(\frac{\rho}{\rho_{0}}\right)+\frac{1+\tau-b}{2}(r \log r-r)
$$


where $\rho_{0}=A^{-1 / 2}$. The optimal order $r=N_{\text {opt }}$ corresponds to the minimum of equation (64), namely

$$
N_{\mathrm{opt}}=\left(\frac{\rho}{\rho_{0}}\right)^{\frac{-2}{1+\tau-b}} .
$$

In the case $\omega_{2} / \omega_{1}=(\sqrt{5}-1) / 2(\tau=1, b=0.3$ i.e. the difference of 1.7 from 2$)$ we find

$$
N_{\mathrm{opt}}=\left(\frac{\rho}{\rho_{0}}\right)^{-1.17} \text {. }
$$

This exponent is close to the exponent found numerically with the remainder criterion (equation (19)). At any rate, we emphasize that equations (59) and (65) represent just average power laws which have meaning only after a sufficient number of steps in the diagram $\left\|R_{r+2}\right\| /\left\|R_{r}\right\|$ versus $r$. In most cases this happens at quite large orders, so that in the actual estimates for $N_{\mathrm{opt}}$ one should find first the function $\lambda_{r}$ and then draw the plateaux and find the abrupt steps in $N_{\text {opt }}$ as described above.

\section{Resonance case}

We now turn our attention to the resonance cases considered numerically in our previous paper. We have a resonance whenever $\omega_{1}: \omega_{2}=n: m$, with $n, m$ integer. The normal form contains kernel terms of the operator $D_{\omega}$. In the resonance case, these are not only terms independent on the angles, but also terms of the form

$$
J_{1}^{\frac{s_{1}}{2}} J_{2}^{\frac{s_{2}}{2}} \exp \left(\mathrm{i}\left(k_{1} \phi_{1}+k_{2} \phi_{2}\right)\right)
$$

where $k_{1}, k_{2} \neq 0$ satisfy the condition

$$
\frac{k_{1}}{k_{2}}=-\frac{m}{n} \text {. }
$$

Thus $Z_{r} \neq 0$ if $r \geqslant n+m$, independent of the parity of $r$.

Consider the cases $n: m=1: 1,1: 3$ and $3: 1$. In these cases $Z_{4}$ contains some Fourier modes depending on the angles. Looking again to equation (27) we find the terms

$$
\chi_{r+4}=-D_{\omega}^{-1}\left\{\chi_{r+2}, Z_{4}\right\}+\cdots=D_{\omega}^{-1}\left\{D_{\omega}^{-1}\left\{\chi_{r}, Z_{4}\right\}, Z_{4}\right\}+\cdots .
$$

But the Poisson bracket of $Z_{4}$ with $\chi_{r}$ introduces now new Fourier modes in $\chi_{r+2}$, different from the Fourier modes of $\chi_{r}$, because the normal form term $Z_{4}$ contains some Fourier modes of order higher than zero. Similarly, new Fourier modes are introduced in $\chi_{r+4}$ by the Poisson bracket of $\chi_{r+2}$ with $Z_{4}$. Therefore, in the resonant case we have non-repetition paths, which appear also in the Lie generating function. The sequence generated by equation (30), for $s=4$, leads now to the recursion (say, for $r$ odd)

$$
\left\|\chi_{r}\right\| \sim \frac{O(r)}{a_{r}}\left\|\chi_{r-2}\right\| \sim \cdots \sim \frac{O(r) O(r-2) \cdots O(3)}{a_{r} a_{r-2} \cdots a_{3}} .
$$

However, there is a lower bound for the denominators $a_{r}, a_{r+2}, \ldots$ This is because at any order the denominator corresponding to a resonant Fourier mode $\exp (\mathrm{i}(k \cdot \phi))$ is equal to zero, i.e. $k \cdot \omega=0$, and such a mode cannot appear in $\chi_{r}$, but it appears only in the normal form. The continued fraction expansion of the rational ratio $\omega_{2} / \omega_{1}$ has a finite number of truncates $q_{1} / p_{1}, q_{2} / p_{2}, \ldots, q_{n} / p_{n}$, where $q_{n} / p_{n}=\omega_{2} / \omega_{1}$. Thus for any divisor $a_{r}$ in the generating function we have

$$
\left|a_{r}\right| \geqslant a=\left|q_{n-1} \omega_{1}-p_{n-1} \omega_{2}\right|
$$


r even

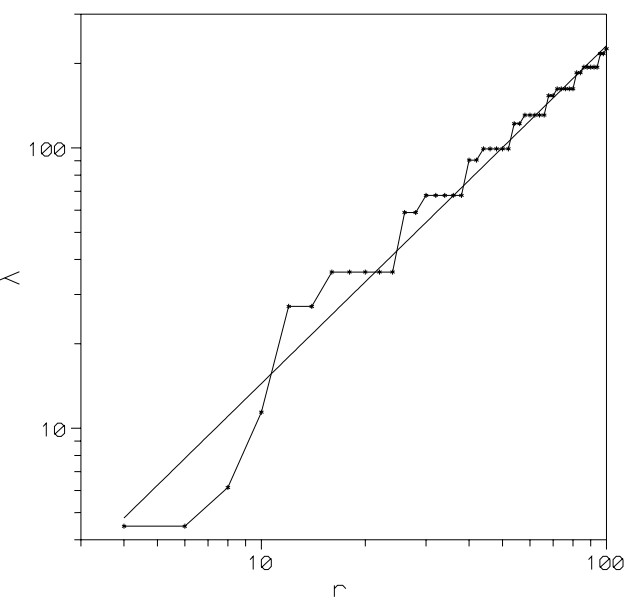

$r$ odd

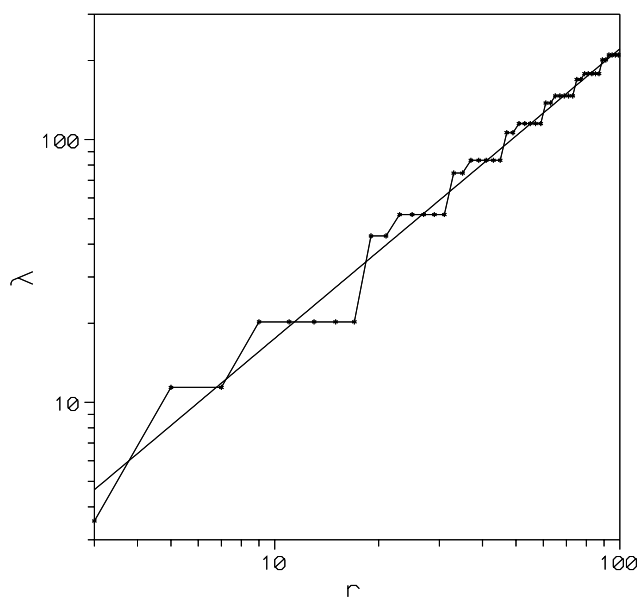

Figure 10. Same as in figure 5, for the frequencies $\omega_{1}=\sqrt{1.6}, \omega_{2}=\sqrt{0.9}$, with frequency ratio $4: 3$. The solid lines represent average power law fittings.

where $a=O(|\omega|)$, and the estimate (69) takes the form

$$
\left\|\chi_{r}\right\| \sim r !^{1 / 2} \text {. }
$$

A similar estimate is found in the case $n: m=1 / 2$ (or $2 / 1)$. In this case the normal form $Z_{3}$ contains some Fourier modes depending on the angles, and these modes act exactly as above for the generating function calculated at every odd order.

On the other hand, if we are in a higher order resonance $n: m$ with $n+m>4$, then, with similar arguments, we find terms in the generating function which grow as $r !^{\frac{1}{n+m-2}}$. For example, if we are in the $5: 1$ resonance, we have terms growing as $r !^{\frac{1}{4}}$ by the sequence

$$
\chi_{r+8}=-D_{\omega}^{-1}\left\{\chi_{r+4}, Z_{6}\right\}+\cdots=D_{\omega}^{-1}\left\{D_{\omega}^{-1}\left\{\chi_{r}, Z_{6}\right\}, Z_{6}\right\}+\cdots .
$$

However, these terms are not the most important. Besides these terms, the terms produced by the sequence given by equation (68) grow exactly as in the non-resonant case, because $Z_{4}$ does not contain terms depending on the angles. Thus the sequence (68) generates terms which grow exactly in the same manner as in the non-resonant case, i.e., they produce a piecewise geometrical growth with a factor $\lambda_{r}$ given by equation (38). If we calculate $\lambda_{r}$ as a function of $r$ in the case of high-order resonances we find that this function behaves as in the non-resonant case, namely it increases by abrupt steps. For example, figure 10 shows $\lambda_{r}$ as a function of $r$ for the resonance $4: 3\left(\omega_{1}^{2}=1.6, \omega_{2}^{2}=0.9\right)$ considered in our previous paper. In this case we can find an average power law

$$
\lambda_{r} \simeq 0.9 r^{1.2} \quad r \text { even, } \quad \lambda_{r} \simeq 1.4 r^{1.1} \quad r \text { odd. }
$$

The power exponent is close to 1 for both even and odd orders. This is expected since we get only an $O(r)$ contribution from the numerator of equation (38).

The plateaux formed by $\lambda_{r}$ (figure 10) are of constant length (equal to $n+m$ ) which makes them look smaller, on a logarithmic scale, as $r$ increases. However, each plateau corresponds to modes the size of which provides a dominant contribution to the series for an interval of values of $r$ much larger than $m+n$. This is due to the delay mechanism. Let $r_{0}$ be the order at which a new step appears in $\lambda_{r}$. Then, the next step appears at $r_{0}^{\prime}=r_{0}+n+m$. Thus we have $\lambda_{r 0} \sim r_{0}$ and $\lambda_{r 0^{\prime}} \sim r_{0}^{\prime}+n+m$. The corresponding Fourier modes grow independently 
and geometrically. They become of equal size after $v$ steps following $r_{0}^{\prime}$, where $v$ satisfies the equation

$$
\left(r_{0}+n+m\right)^{v} \simeq r_{0}^{v+n+m}
$$

which yields the estimate

$$
v \simeq \frac{(n+m) \log r_{0}}{\log \left(\frac{r_{0}+n+m}{r_{0}}\right)} .
$$

In the limit $r_{0} \gg n+m$ the above equation tends to

$$
v \simeq r_{0} \log r_{0} .
$$

This formula is similar to equation (56), but with $r_{0}$ in the numerator instead of the difference $r_{0}^{\prime}-r_{0}$. This fact means that the delay increases as $r_{0}$ increases. Thus, the factor $\lambda_{r 0}$ is dominant for at least $r_{0}$ double steps, which means that the growth of the Fourier modes in the generating function with this particular factor is $O\left(r^{r / 2}\right) \sim r !^{1 / 2}$ on average (but the true function has abrupt steps). For high-order resonances, this growth is much more important than the growth $r !^{\frac{1}{n+m-2}}$ induced by non-repetition paths.

The conclusion is that in the resonant case the generating function grows on average as $\left\|\chi_{r}\right\| \sim r !^{1 / 2}$. However, the growth is by abrupt steps in the case of high-order resonances $n: m, n+m>4$, while it is rather smooth in the case of low-order resonances $n+m \leqslant 4$. This explains the results found in our previous paper, namely that $N_{\text {opt }}$ depends smoothly on $\rho$ in the $1: 1$ resonance, but the dependence is by abrupt steps in a higher order $(4: 3)$ resonance.

\section{Conclusions}

We study the asymptotic properties of formal integral series in the neighbourhood of an elliptic equilibrium in 2 DOF Hamiltonian nonlinear dynamical systems. In particular, we examine in detail the way by which the accumulation of small divisors affects the size of the remainder and the optimal order of truncation of formal series. Our study is both numerical by calculating the series up to a high order, and analytical, by making appropriate estimates in terms of both the Lie generating function series and the formal integral series produced by a direct method. The main conclusions are the following:

(1) In the non-resonant case, the overall behaviour of the perturbation series is essentially determined by repetition of Fourier terms. Under the action of a generating function of order $r$ no new Fourier modes are generated up to order $2 r$, while the modes appearing in the generating function are repeated at every successive order. This creates a repetition path of period 1. New Fourier modes at any order $r$ are introduced after $r-1$ transformations only by the terms of order $r$ of the Hamiltonian.

(2) The repetition paths generate sequences of terms that grow independently and geometrically. The growth of the overall size of the series is determined by the fastest growing Fourier mode. The geometrical factor $\lambda_{r}$ of the fastest growing mode may be explicitly evaluated, and is given by equation (38). The factor $\lambda_{r}$ increases by abrupt steps at the orders $r$ where new diophantine divisors appear. Thus, the behaviour of $\lambda_{r}$ is determined essentially by the number theoretical properties of the frequency ratio $\omega_{2} / \omega_{1}$. Repetition paths of period greater than 1 do appear, as well as modes that are generated by non-repetition paths. However, these modes are dominated by the repetition paths of period 1. 
(3) The same phenomena occur also in the series generated by a direct construction of first integrals. Thus, the formal series produced by the direct method grows in the same way as the series produced by the normal form with Lie series, although the two series are not formally equivalent.

(4) At an order where a new mode appears with a smaller denominator, its size is smaller than the size of the current dominant term. Thus, the new mode becomes dominant only after a certain number of steps. We call this phenomenon a 'delay' and provide analytical estimates for it (section 2.3). There are also transient intervals of values of $r$ in which two modes have about equal size. Such intervals produce an 'inversion' phenomenon, namely, a third mode with larger divisor may become dominant for a transient number of iterations.

(5) The behaviour of the function $\lambda_{r}$ is related to the behaviour of the function $N_{\text {opt }}(\rho)$, i.e. of the optimal order of truncation as a function of the distance from the elliptic equilibrium. In particular, the plateaux of the function $\lambda_{r}$, together with the delay estimates, provide an explanation for the plateaux of the function $N_{\text {opt }}(\rho)$. In the case where many plateaux appear, an average power law of the form $N_{\mathrm{opt}}=O\left(\rho^{\frac{-2}{1+\tau-b}}\right)$ is found, where $\tau$ is a diophantine constant (see equation (2)) and $b$ is a delay constant, $b<1$. This law is found theoretically and it agrees well with the law found numerically by calculating the remainder of the formal series up to order 120.

(6) Similar effects, as in the non-resonant case, appear also in the resonant case for resonances $n: m$ with $n+m>4$. The formal series in such resonances grow in the same stepwise manner as the series in non-resonant cases. This is because the function $\lambda_{r}$ increases by abrupt steps, and the repetition modes created by the non-resonant terms of the normal form can be shown to have a size larger than the size of the modes created by the resonant part of the normal form. However, the reverse is true in low-order resonances $n+m \leqslant 4$. This explains the differences found numerically in our previous paper (Contopoulos et al $2003)$ for the $1: 1$ resonance and for a higher order $(4: 3)$ resonance.

\section{Acknowledgment}

This research was supported in part by the Academy of Athens grant 417/2001.

\section{References}

Arnold V I 1985 Encyclopedia of Dynamical Systems vol 3 (Berlin: Springer) Birkhoff G D 1927 Dynamical Systems (Providence, RI: American Mathematical Society)

Boccaletti D and Pucacco G 1999 Theory of Orbits vol 2 (Berlin: Springer)

Cherry T M 1924 Proc. Camb. Phil. Soc. 22325

Cherry T M 1924 Proc. Camb. Phil. Soc. 22510

Contopoulos G 1960 Z. Astrophys. 49273

Contopoulos G 1963 Astron. J. 68763

Contopoulos G 2002 Order and Chaos in Dynamical Astronomy (Berlin: Springer)

Contopoulos G and Moutsoulas M 1965 Astron. J. 70817

Contopoulos G, Efthymiopoulos C and Giorgilli A 2003 J. Phys. A: Math. Gen. 368639

Deprit A 1969 Celest. Mech. 112

Eckhardt B 1986 J. Phys. A: Math. Gen. 192961

Fassò F, Guzzo M and Benettin G 1998 Commun. Math. Phys. 197347

Giorgilli A 1979 Comput. Phys. Commun. 16331

Giorgilli A 1988 Ann. Inst. H Poincaré 48423

Giorgilli A 1999 Hamiltonian Systems with Three or More Degrees of Freedom ed C Simo (Dordrecht: Kluwer)

Gustavson F 1966 Astron. J. 71670 
Haller G 1999 Chaos Near Resonance (Berlin: Springer)

Hori G I 1966 Publ. Astron. Soc. Japan 18287

Kaluza M and Robnik M 1992 J. Phys. A: Math. Gen. 255311

Lochak P 1992 Russ. Math. Surv. 4757

Morbidelli A and Giorgilli A 1997 Physica D 102195

Nekhoroshev N N 1977 Russ. Math. Surv. 321

Niederman L 1998 Nonlinearity 111465

Poincaré H 1892 Les Méthodes Nouvelles de la Mécanique Céleste (Paris: Gauthier-Villars)

Servizi G, Turchetti G, Benettin G and Giorgilli A 1983 Phys. Lett. A 9511

Siegel C L 1941 Ann. Math. 42806

Whittaker E T 1916 Proc. R. Soc. Edinburgh 3795

Whittaker E T 1937 A Treatise on the Analytical Dynamics of Particles and Rigid Bodies (Cambridge: Cambridge University Press)

Wood W R and Ali M K 1987 J. Phys. A: Math. Gen. 20351 